\title{
SPIN SCISSORS MODE AND THE FINE STRUCTURE OF M1 STATES IN NUCLEI
}

\author{
E.B. Balbutsev, I.V. Molodtsova \\ Joint Institute for Nuclear Research, 141980 Dubna, Moscow Region, Russia \\ P. Schuck \\ Institut de Physique Nucleaire, Orsay Cedex 91406, France
}

\begin{abstract}
The coupled dynamics of low lying modes, including the scissors mode, and various giant quadrupole resonances are studied with the help of the Wigner Function Moments method generalized to take into account spin degrees of freedom. Equations of motion for collective variables are derived on the basis of Time Dependent Hartree-Fock equations in the model of harmonic oscillator with spin orbital mean field potential plus quadrupolequadrupole residual interaction. Introducing spin allows one to consider new types of nuclear collective motion where the nucleons with spin 'up' oscillate against nucleons with spin 'down'.
\end{abstract}

\section{Introduction}

The nuclear scissors mode was predicted $[1,2,3,4]$ as the collective motion of two types of nucleons - the protons undergo counter-rotational vibrations with respect to the neutrons. However, its collectivity turned out so small that it was even discussed whether [5] this mode is really collective. Pure phenomenological models (such as, for example, two rotors model [6]) could not help clarifying this question. The results of RPA calculations [5] were in qualitative agreement with the experiment, an indication in favour of non collectivity. The final conclusion was that the scissors mode is [7] "weakly collective, but strong on the single-particle scale". As a result [7]: "The weekly collective scissors mode excitation has become an ideal test of models - especially microscopic models - of nuclear vibrations. Most models are usually calibrated to reproduce properties of strongly collective excitations (e.g. of $J^{\pi}=2^{+}$or $3^{-}$states, 
giant resonances, ...). Weekly-collective phenomena, however, force the models to make genuine predictions and the fact that the transitions in question are strong on the single-particle scale makes it impossible to dismiss failures as a mere detail, especially in the light of the overwhelming experimental evidence for them in many nuclei $[8,9]$."

The Wigner Function Moments (WFM) or phase space moments method turns out to be very useful in this situation. On the one hand it is a pure microscopic method, because it is based on the Time Dependent Hartree-Fock (TDHF) equation. On the other hand the method works with average values (moments) of operators which have a direct relation to the considered phenomenon. That makes it an ideal instrument to describe the basic characteristics (energies and excitation probabilities) of collective excitations such as, in particular, the scissors mode. Our investigations have shown that already the minimal set of collective variables, i.e. phase space moments up to quadratic order, is sufficient to reproduce the most important properties of the scissors mode: its inevitable coexistence with the IsoVector Giant Quadrupole Resonance (IVGQR) implying a deformation of the Fermi surface. Also there is no doubt about its collectiveness, because the principal variables generating this mode (angular momenta of protons and neutrons) are collective being the respective operators averaged over all protons and neutrons.

Further developments of the Wigner Function Moments method, namely, the switch from TDHF to TDHFB equations, i.e. taking into account pair correlations, allowed us to improve considerably the quantitative description of the scissors mode [10, 11]: for rare earth nuclei the energies are reproduced with $\sim 10 \%$ accuracy and B(M1) factors were reduced about two times. However, they remain about two times too high with respect to experiment. The reason of the last discrepancy is probably hidden in the spin degrees of freedom, which were so far ignored by WFM method. One can not exclude, that due to spin dependent interactions some part of the force of M1 transitions is shifted to the energy region of 5-10 MeV, where $1^{+}$resonance of the spin nature is observed. The generalization of the WFM method to take into account spin degrees of freedom is the goal of this paper. In a first step, we include in the consideration only the spin orbital interaction, as the most important one among all possible spin dependent interactions because it enters into the mean field. This allows us to understand the structure 
of necessary modifications of the method avoiding too cumbersome calculations. In this way it becomes clear already on the level of formulation of the equations of motion for new collective variables, that we are faced with a new type of collective motion, namely the spin scissors mode. It turns out that the experimentally observed group of peaks in the energy interval 2-4 MeV corresponds to two different types of motion: "standard" scissors and this new kind of mode, i.e. spin scissors.

The paper is organized as follows. In Sec. 2 the TDHF equations for 4-component density matrix are formulated and their Wigner transform is found. In Sec. 3 the spin structure of the density matrix and the model Hamiltonian are studied. In Sec. 4 the collective variables are defined and the respective dynamical equations are derived. In Sec. 5 the procedure of calculation of excitation probabilities is recalled and the results of calculations of energies, B(M1) and $\mathrm{B}(\mathrm{E} 2)$ factors are discussed. Last remarks and the outlook are given in the conclusion section.

\section{TDHF equation with spin}

In this section we will consider the TDHF equation in coordinate space keeping all the spin indices. The TDHF equation in operator formulation reads [13]

$$
i \hbar \dot{\hat{\rho}}=[\hat{h}, \hat{\rho}]
$$

or in matrix form

$$
\begin{aligned}
& i \hbar<\mathbf{r}, s|\dot{\hat{\rho}}| \mathbf{r}^{\prime \prime}, s^{\prime \prime}>= \\
& \sum_{s^{\prime}} \int d^{3} r^{\prime}\left(<\mathbf{r}, s|\hat{h}| \mathbf{r}^{\prime}, s^{\prime}><\mathbf{r}^{\prime}, s^{\prime}|\hat{\rho}| \mathbf{r}^{\prime \prime}, s^{\prime \prime}>-<\mathbf{r}, s|\hat{\rho}| \mathbf{r}^{\prime}, s^{\prime}><\mathbf{r}^{\prime}, s^{\prime}|\hat{h}| \mathbf{r}^{\prime \prime}, s^{\prime \prime}>\right) .
\end{aligned}
$$

We do not specify the isospin indices in order to make the formulae more transparent. They will be re-introduced at the end. The set of TDHF equations (2) with specified spin indices reads

$$
\begin{array}{r}
i \hbar<\mathbf{r}|\dot{\hat{\rho}}| \mathbf{r}^{\prime \prime}>^{\uparrow \uparrow}=\int d^{3} r^{\prime}\left(<\mathbf{r}|\hat{h}| \mathbf{r}^{\prime}>^{\uparrow \uparrow}<\mathbf{r}^{\prime}|\hat{\rho}| \mathbf{r}^{\prime \prime}>^{\uparrow \uparrow}-<\mathbf{r}|\hat{\rho}| \mathbf{r}^{\prime}>^{\uparrow \uparrow}<\mathbf{r}^{\prime}|\hat{h}| \mathbf{r}^{\prime \prime}>^{\uparrow \uparrow}\right. \\
\left.+<\mathbf{r}|\hat{h}| \mathbf{r}^{\prime}>^{\uparrow \downarrow}<\mathbf{r}^{\prime}|\hat{\rho}| \mathbf{r}^{\prime \prime}>^{\downarrow \uparrow}-<\mathbf{r}|\hat{\rho}| \mathbf{r}^{\prime}>^{\uparrow \downarrow}<\mathbf{r}^{\prime}|\hat{h}| \mathbf{r}^{\prime \prime}>^{\downarrow \uparrow}\right), \\
i \hbar<\mathbf{r}|\dot{\hat{\rho}}| \mathbf{r}^{\prime \prime}>^{\uparrow \downarrow}=\int d^{3} r^{\prime}\left(<\mathbf{r}|\hat{h}| \mathbf{r}^{\prime}>^{\uparrow \uparrow}<\mathbf{r}^{\prime}|\hat{\rho}| \mathbf{r}^{\prime \prime}>^{\uparrow \downarrow}-<\mathbf{r}|\hat{\rho}| \mathbf{r}^{\prime}>^{\uparrow \uparrow}<\mathbf{r}^{\prime}|\hat{h}| \mathbf{r}^{\prime \prime}>^{\uparrow \downarrow}\right.
\end{array}
$$




$$
\begin{aligned}
& \left.+<\mathbf{r}|\hat{h}| \mathbf{r}^{\prime}>^{\uparrow \downarrow}<\mathbf{r}^{\prime}|\hat{\rho}| \mathbf{r}^{\prime \prime}>^{\downarrow \downarrow}-<\mathbf{r}|\hat{\rho}| \mathbf{r}^{\prime}>^{\uparrow \downarrow}<\mathbf{r}^{\prime}|\hat{h}| \mathbf{r}^{\prime \prime}>^{\downarrow \downarrow}\right), \\
& i \hbar<\mathbf{r}|\dot{\hat{\rho}}| \mathbf{r}^{\prime \prime}>^{\downarrow \uparrow}=\int d^{3} r^{\prime}\left(<\mathbf{r}|\hat{h}| \mathbf{r}^{\prime}>^{\downarrow \uparrow}<\mathbf{r}^{\prime}|\hat{\rho}| \mathbf{r}^{\prime \prime}>^{\uparrow \uparrow}-<\mathbf{r}|\hat{\rho}| \mathbf{r}^{\prime}>^{\downarrow \uparrow}<\mathbf{r}^{\prime}|\hat{h}| \mathbf{r}^{\prime \prime}>^{\uparrow \uparrow}\right. \\
& \left.+<\mathbf{r}|\hat{h}| \mathbf{r}^{\prime}>^{\downarrow}<\mathbf{r}^{\prime}|\hat{\rho}| \mathbf{r}^{\prime \prime}>^{\downarrow \uparrow}-<\mathbf{r}|\hat{\rho}| \mathbf{r}^{\prime}>^{\downarrow}<\mathbf{r}^{\prime}|\hat{h}| \mathbf{r}^{\prime \prime}>^{\downarrow \uparrow}\right), \\
& i \hbar<\mathbf{r}|\dot{\hat{\rho}}| \mathbf{r}^{\prime \prime}>^{\downarrow}=\int d^{3} r^{\prime}\left(<\mathbf{r}|\hat{h}| \mathbf{r}^{\prime}>^{\downarrow \uparrow}<\mathbf{r}^{\prime}|\hat{\rho}| \mathbf{r}^{\prime \prime}>^{\uparrow \downarrow}-<\mathbf{r}|\hat{\rho}| \mathbf{r}^{\prime}>^{\downarrow \uparrow}<\mathbf{r}^{\prime}|\hat{h}| \mathbf{r}^{\prime \prime}>^{\uparrow \downarrow}\right. \\
& \left.+<\mathbf{r}|\hat{h}| \mathbf{r}^{\prime}>^{\downarrow}<\mathbf{r}^{\prime}|\hat{\rho}| \mathbf{r}^{\prime \prime}>^{\downarrow}-<\mathbf{r}|\hat{\rho}| \mathbf{r}^{\prime}>^{\downarrow}<\mathbf{r}^{\prime}|\hat{h}| \mathbf{r}^{\prime \prime}>^{\downarrow}\right)
\end{aligned}
$$

with the conventional notation

$$
\uparrow \text { for } s=\frac{1}{2} \text { and } \downarrow \text { for } s=-\frac{1}{2} .
$$

These equations will be solved by the method of phase space (or Wigner function) moments. To this end we will rewrite the expression (3) with the help of Wigner transformation [13].

\subsection{Wigner transformation}

The relevant mathematical details can be found in Appendix. To make the formulae more transparent we will not write out the coordinate dependence $(\mathbf{r}, \mathbf{p})$ of the functions. The Wigner transform of (3) can be written as

$$
\begin{aligned}
i \hbar \dot{f}^{\uparrow \uparrow}= & i \hbar\left\{h^{\uparrow \uparrow}, f^{\uparrow \uparrow}\right\}+h^{\uparrow \downarrow} f^{\downarrow \uparrow}-f^{\uparrow \downarrow} h^{\downarrow \uparrow}+\frac{i \hbar}{2}\left\{h^{\uparrow \downarrow}, f^{\downarrow \uparrow}\right\}-\frac{i \hbar}{2}\left\{f^{\uparrow \downarrow}, h^{\downarrow \uparrow}\right\} \\
& -\frac{\hbar^{2}}{8}\left\{\left\{h^{\uparrow \downarrow}, f^{\downarrow \uparrow}\right\}\right\}+\frac{\hbar^{2}}{8}\left\{\left\{f^{\uparrow \downarrow}, h^{\downarrow \uparrow}\right\}\right\}+\ldots, \\
i \hbar \dot{f}^{\uparrow \downarrow}= & f^{\uparrow \downarrow}\left(h^{\uparrow \uparrow}-h^{\downarrow \downarrow}\right)+\frac{i \hbar}{2}\left\{\left(h^{\uparrow \uparrow}+h^{\downarrow \downarrow}\right), f^{\uparrow \downarrow}\right\}-\frac{\hbar^{2}}{8}\left\{\left\{\left(h^{\uparrow \uparrow}-h^{\downarrow \downarrow}\right), f^{\uparrow \downarrow}\right\}\right\} \\
& -h^{\uparrow \downarrow}\left(f^{\uparrow \uparrow}-f^{\downarrow \downarrow}\right)+\frac{i \hbar}{2}\left\{h^{\uparrow \downarrow},\left(f^{\uparrow \uparrow}+f^{\downarrow \downarrow}\right)\right\}+\frac{\hbar^{2}}{8}\left\{\left\{h^{\uparrow \downarrow},\left(f^{\uparrow \uparrow}-f^{\downarrow \downarrow}\right)\right\}\right\}+\ldots
\end{aligned}
$$

where the functions $h, f$ are the Wigner transforms of $\hat{h}, \hat{\rho}$ respectively, $\{f, g\}$ is the Poisson bracket of the functions $f(\mathbf{r}, \mathbf{p})$ and $g(\mathbf{r}, \mathbf{p})$ and $\{\{f, g\}\}$ is their double Poisson bracket; the dots stand for terms proportional to higher powers of $\hbar$. Two more equations are obtained by the obvious change of arrows $\uparrow \leftrightarrow \downarrow$.

It is useful to rewrite the above equations in terms of functions $f^{+}=f^{\uparrow \uparrow}+f^{\downarrow \downarrow}, f^{-}=f^{\uparrow \uparrow}-f^{\downarrow \downarrow}$. By analogy with isoscalar $f^{\mathrm{n}}+f^{\mathrm{p}}$ and isovector $f^{\mathrm{n}}-f^{\mathrm{p}}$ functions one can name the functions 
$f^{+}$and $f^{-}$as spin-scalar and spin-vector ones respectively. We have:

$$
\begin{aligned}
i \hbar \dot{f}^{+}= & \frac{i \hbar}{2}\left\{h^{+}, f^{+}\right\}+\frac{i \hbar}{2}\left\{h^{-}, f^{-}\right\}+i \hbar\left\{h^{\uparrow \downarrow}, f^{d}\right\}+i \hbar\left\{h^{\downarrow \uparrow}, f^{u}\right\}+\ldots, \\
i \hbar \dot{f}^{-}= & \frac{i \hbar}{2}\left\{h^{+}, f^{-}\right\}+\frac{i \hbar}{2}\left\{h^{-}, f^{+}\right\}-2 h^{\downarrow \uparrow} f^{u}+2 h^{\uparrow \downarrow} f^{d} \\
& +\frac{\hbar^{2}}{4}\left\{\left\{h^{\downarrow \uparrow}, f^{u}\right\}\right\}-\frac{\hbar^{2}}{4}\left\{\left\{h^{\uparrow \downarrow}, f^{d}\right\}\right\}+\ldots, \\
i \hbar \dot{f}^{u}= & -h^{\uparrow \downarrow} f^{-}+h^{-} f^{u}+\frac{i \hbar}{2}\left\{h^{\uparrow \downarrow}, f^{+}\right\}+\frac{i \hbar}{2}\left\{h^{+}, f^{u}\right\} \\
& +\frac{\hbar^{2}}{8}\left\{\left\{h^{\uparrow \downarrow}, f^{-}\right\}\right\}-\frac{\hbar^{2}}{8}\left\{\left\{h^{-}, f^{u}\right\}\right\}+\ldots, \\
i \hbar \dot{f}^{d}= & h^{\downarrow \uparrow} f^{-}-h^{-} f^{d}+\frac{i \hbar}{2}\left\{h^{\downarrow \uparrow}, f^{+}\right\}+\frac{i \hbar}{2}\left\{h^{+}, f^{d}\right\} \\
& -\frac{\hbar^{2}}{8}\left\{\left\{h^{\downarrow \uparrow}, f^{-}\right\}\right\}+\frac{\hbar^{2}}{8}\left\{\left\{h^{-}, f^{d}\right\}\right\}+\ldots,
\end{aligned}
$$

where $f^{u}=f^{\uparrow \downarrow}, f^{d}=f^{\downarrow \uparrow}, h^{ \pm}=h^{\uparrow \uparrow} \pm h^{\downarrow \downarrow}$.

\section{Model Hamiltonian}

The microscopic Hamiltonian of the model, harmonic oscillator with spin orbital interaction plus separable quadrupole-quadrupole residual interaction is given by

$$
\begin{aligned}
H=\sum_{i=1}^{A}\left[\frac{\hat{\mathbf{p}}_{i}^{2}}{2 m}+\right. & \left.\frac{1}{2} m \omega^{2} \mathbf{r}_{i}^{2}-\eta\left(\mathbf{r}_{i}\right) \hat{\mathbf{l}}_{i} \hat{\mathbf{S}}_{i}\right]+\bar{\kappa} \sum_{\mu=-2}^{2}(-1)^{\mu} \sum_{i}^{Z} \sum_{j}^{N} q_{2-\mu}\left(\mathbf{r}_{i}\right) q_{2 \mu}\left(\mathbf{r}_{j}\right) \\
& +\frac{1}{2} \kappa \sum_{\mu=-2}^{2}(-1)^{\mu}\left\{\sum_{i \neq j}^{Z} q_{2-\mu}\left(\mathbf{r}_{i}\right) q_{2 \mu}\left(\mathbf{r}_{j}\right)+\sum_{i \neq j}^{N} q_{2-\mu}\left(\mathbf{r}_{i}\right) q_{2 \mu}\left(\mathbf{r}_{j}\right)\right\},
\end{aligned}
$$

where $N$ and $Z$ are the numbers of neutrons and protons, respectively. The quadrupole operator $q_{2 \mu}$ can be written as the tensor product: $q_{2 \mu}(\mathbf{r})=\sqrt{16 \pi / 5} r^{2} Y_{2 \mu}(\theta, \phi)=\sqrt{6}\{r \otimes r\}_{2 \mu}$, where

$$
\{r \otimes r\}_{\lambda \mu}=\sum_{\sigma, \nu} C_{1 \sigma, 1 \nu}^{\lambda \mu} r_{\sigma} r_{\nu}
$$

$r_{-1}, r_{0}, r_{1}$ are cyclic coordinates [14] and $C_{1 \sigma, 1 \nu}^{\lambda \mu}$ is the Clebsch-Gordan coefficient. The mean field potential for protons (or neutrons) is

$$
V^{\tau}=\frac{1}{2} m \omega^{2} r^{2}+\sum_{\mu}(-1)^{\mu} Z_{2-\mu}^{\tau+}\{r \otimes r\}_{2 \mu}-\eta(\mathbf{r}) \hat{\mathbf{l}} \hat{\mathbf{S}}
$$


Here

$$
\begin{gathered}
Z_{2 \mu}^{\mathrm{n}+}=\chi R_{2 \mu}^{\mathrm{n}+}+\bar{\chi} R_{2 \mu}^{\mathrm{p}+}, \quad Z_{2 \mu}^{\mathrm{p}+}=\chi R_{2 \mu}^{\mathrm{p}+}+\bar{\chi} R_{2 \mu}^{\mathrm{n}+}, \quad \chi=6 \kappa, \quad \bar{\chi}=6 \bar{\kappa} \\
R_{\lambda \mu}^{\tau+}(t)=\int d\{\mathbf{p}, \mathbf{r}\} r_{\lambda \mu}^{2} f^{\tau+}(\mathbf{r}, \mathbf{p}, t)
\end{gathered}
$$

with $\int d\{\mathbf{p}, \mathbf{r}\} \equiv(2 \pi \hbar)^{-3} \int d^{3} p \int d^{3} r$.

\subsection{Spin structure}

Let us clarify the spin structure of the density matrix and the Hamiltonian.

\subsubsection{Density matrix}

As an example we consider the spherical case:

$$
\begin{aligned}
& \psi_{n l j m}(\mathbf{r}, s) \equiv<n l j m \mid \mathbf{r}, s>=\mathcal{R}_{n l}(r) \sum_{\Lambda, \sigma} C_{l \Lambda, \frac{1}{2} \sigma}^{j m} Y_{l \Lambda}(\theta, \phi) \chi_{\frac{1}{2} \sigma}(s), \\
& \psi_{n l j m}^{*}(\mathbf{r}, s) \equiv<\mathbf{r}, s \mid n l j m>=\mathcal{R}_{n l}(r) \sum_{\Lambda, \sigma} C_{l \Lambda, \frac{1}{2} \sigma}^{j m} Y_{l \Lambda}^{*}(\theta, \phi) \chi_{\frac{1}{2} \sigma}^{\dagger}(s) .
\end{aligned}
$$

Spin functions are defined as

$$
\chi_{\frac{1}{2} \frac{1}{2}}(s)=\left(\begin{array}{c}
\delta_{s, \frac{1}{2}} \\
0
\end{array}\right) \equiv \chi_{\uparrow}(s), \quad \chi_{\frac{1}{2}-\frac{1}{2}}(s)=\left(\begin{array}{c}
0 \\
\delta_{s,-\frac{1}{2}}
\end{array}\right) \equiv \chi_{\downarrow}(s), \quad \chi_{\frac{1}{2} \frac{1}{2}}^{\dagger}(s)=\left(\delta_{s, \frac{1}{2}}, 0\right) .
$$

The density matrix reads $[13]$

$$
\begin{aligned}
<\mathbf{r}, s|\hat{\rho}| \mathbf{r}^{\prime}, s^{\prime}>= & \sum_{n l j m} v_{n l j}^{2}<\mathbf{r}, s|n l j m><n l j m| \mathbf{r}^{\prime}, s^{\prime}> \\
= & \sum_{n l j m} v_{n l j}^{2} \mathcal{R}_{n l}(r) \mathcal{R}_{n l}\left(r^{\prime}\right) \sum_{\sigma_{1}, \sigma_{2}} C_{l m-\sigma_{1}, \frac{1}{2} \sigma_{1}}^{j m} C_{l m-\sigma_{2}, \frac{1}{2} \sigma_{2}}^{j m} \\
& Y_{l m-\sigma_{1}}(\theta, \phi) Y_{l m-\sigma_{2}}^{*}\left(\theta^{\prime}, \phi^{\prime}\right) \chi_{\frac{1}{2} \sigma_{1}}(s) \chi_{\frac{1}{2} \sigma_{2}}^{\dagger}\left(s^{\prime}\right),
\end{aligned}
$$

where $v_{n l j}^{2}$ are the occupation numbers of level $n l j$. According to the definition (10) the spin structure of the density matrix is

$$
<\mathbf{r}, s|\hat{\rho}| \mathbf{r}^{\prime}, s^{\prime}>=\rho\left(\mathbf{r} s, \mathbf{r}^{\prime} s^{\prime}\right)\left(\begin{array}{ll}
\delta_{s \uparrow} \delta_{s^{\prime} \uparrow} & \delta_{s \uparrow} \delta_{s^{\prime} \downarrow} \\
\delta_{s \downarrow} \delta_{s^{\prime} \uparrow} & \delta_{s \downarrow} \delta_{s^{\prime} \downarrow}
\end{array}\right),
$$

where

$$
\rho\left(\mathbf{r} s, \mathbf{r}^{\prime} s^{\prime}\right)=\sum_{n l j m} v_{n l j}^{2} \phi_{n l j m}(\mathbf{r}, s) \phi_{n l j m}^{*}\left(\mathbf{r}^{\prime}, s^{\prime}\right)
$$


and

$$
\phi_{n l j m}(\mathbf{r}, s)=\mathcal{R}_{n l}(r) C_{l m-s, \frac{1}{2} s}^{j m} Y_{l m-s}(\theta, \phi) .
$$

\subsection{2 l-s Hamiltonian}

Written in cyclic coordinates, the spin orbit part of the Hamiltonian reads

$$
\hat{h}_{l s}=-\eta(\mathbf{r}) \sum_{\mu=-1}^{1}(-)^{\mu} \hat{l}_{\mu} \hat{S}_{-\mu}=-\eta(\mathbf{r})\left(\begin{array}{cc}
\hat{l}_{0} \frac{\hbar}{2} & \hat{l}_{-1} \frac{\hbar}{\sqrt{2}} \\
-\hat{l}_{1} \frac{\hbar}{\sqrt{2}} & -\hat{l}_{0} \frac{\hbar}{2}
\end{array}\right),
$$

where [14]

$$
\begin{gathered}
\hat{l}_{1}=\hbar\left(r_{0} \nabla_{1}-r_{1} \nabla_{0}\right)=\frac{\hbar}{\sqrt{2}}\left[x \nabla_{z}-z \nabla_{x}+i\left(y \nabla_{z}-z \nabla_{y}\right)\right]=-\frac{1}{\sqrt{2}}\left(\hat{l}_{x}+i \hat{l}_{y}\right), \\
\hat{l}_{0}=\hbar\left(r_{-1} \nabla_{1}-r_{1} \nabla_{-1}\right)=-i \hbar\left(x \nabla_{y}-y \nabla_{x}\right)=\hat{l}_{z}, \\
\hat{l}_{-1}=\hbar\left(r_{-1} \nabla_{0}-r_{0} \nabla_{-1}\right)=\frac{\hbar}{\sqrt{2}}\left[x \nabla_{z}-z \nabla_{x}-i\left(y \nabla_{z}-z \nabla_{y}\right)\right]=\frac{1}{\sqrt{2}}\left(\hat{l}_{x}-i \hat{l}_{y}\right), \\
\hat{S}_{1}=-\frac{\hbar}{\sqrt{2}}\left(\begin{array}{ll}
0 & 1 \\
0 & 0
\end{array}\right), \quad \hat{S}_{0}=\frac{\hbar}{2}\left(\begin{array}{rr}
1 & 0 \\
0 & -1
\end{array}\right), \quad \hat{S}_{-1}=\frac{\hbar}{\sqrt{2}}\left(\begin{array}{ll}
0 & 0 \\
1 & 0
\end{array}\right),
\end{gathered}
$$

The matrix elements of $\hat{h}_{l s}$ in the configuration space are:

$$
\begin{array}{r}
<\nu\left|\hat{h}_{l s}\right| \nu^{\prime}>=\sum_{s, s^{\prime}} \int d^{3} r<\nu\left|\mathbf{r}, s>h_{l s}<\mathbf{r}, s^{\prime}\right| \nu^{\prime}> \\
=-\sum_{s, s^{\prime}} \int d^{3} r \mathcal{R}_{n l}(r) \sum_{\Lambda, \sigma} C_{l \Lambda, \frac{1}{2} \sigma}^{j m} Y_{l \Lambda}^{*}(\theta, \phi) \chi_{\frac{1}{2} \sigma}^{\dagger}(s) \\
\eta(\mathbf{r}) \sum_{\mu}(-)^{\mu} \hat{l}_{\mu}(\mathbf{r}) \hat{S}_{-\mu} \mathcal{R}_{n^{\prime} l^{\prime}}(r) \sum_{\Lambda^{\prime}, \sigma^{\prime}} C_{l^{\prime} \Lambda^{\prime}, \frac{1}{2} \sigma^{\prime}}^{j^{\prime} m^{\prime}} Y_{l^{\prime} \Lambda^{\prime}}(\theta, \phi) \chi_{\frac{1}{2} \sigma^{\prime}}\left(s^{\prime}\right),
\end{array}
$$

where $\nu \equiv n, l, j, m$. Taking into account, that

$$
\begin{gathered}
\chi_{\uparrow}^{\dagger}(s) \hat{S}_{1} \chi_{\uparrow}\left(s^{\prime}\right)=0, \quad \chi_{\uparrow}^{\dagger}(s) \hat{S}_{-1} \chi_{\uparrow}\left(s^{\prime}\right)=0, \quad \chi_{\uparrow}^{\dagger}(s) \hat{S}_{0} \chi_{\uparrow}\left(s^{\prime}\right)=\frac{\hbar}{2} \delta_{s \frac{1}{2}} \delta_{s^{\prime} \frac{1}{2}}, \\
\chi_{\downarrow}^{\dagger}(s) \hat{S}_{1} \chi_{\downarrow}\left(s^{\prime}\right)=0, \quad \chi_{\downarrow}^{\dagger}(s) \hat{S}_{-1} \chi_{\downarrow}\left(s^{\prime}\right)=0, \quad \chi_{\downarrow}^{\dagger}(s) \hat{S}_{0} \chi_{\downarrow}\left(s^{\prime}\right)=-\frac{\hbar}{2} \delta_{s-\frac{1}{2}} \delta_{s^{\prime}-\frac{1}{2}}, \\
\chi_{\uparrow}^{\dagger}(s) \hat{S}_{1} \chi_{\downarrow}\left(s^{\prime}\right)=-\frac{\hbar}{\sqrt{2}} \delta_{s \frac{1}{2}} \delta_{s^{\prime}-\frac{1}{2}}, \quad \chi_{\uparrow}^{\dagger}(s) \hat{S}_{-1} \chi_{\downarrow}\left(s^{\prime}\right)=0, \quad \chi_{\uparrow}^{\dagger}(s) \hat{S}_{0} \chi_{\downarrow}\left(s^{\prime}\right)=0,
\end{gathered}
$$




$$
\chi_{\downarrow}^{\dagger}(s) \hat{S}_{1} \chi_{\uparrow}\left(s^{\prime}\right)=0, \quad \chi_{\downarrow}^{\dagger}(s) \hat{S}_{-1} \chi_{\uparrow}\left(s^{\prime}\right)=\frac{\hbar}{\sqrt{2}} \delta_{s-\frac{1}{2}} \delta_{s^{\prime} \frac{1}{2}}, \quad \chi_{\downarrow}^{\dagger}(s) \hat{S}_{0} \chi_{\uparrow}\left(s^{\prime}\right)=0,
$$

we find:

$$
\begin{gathered}
<\nu\left|\hat{h}_{l s}\right| \nu^{\prime}>=-\sum_{s, s^{\prime}} \int d^{3} r \sum_{\Lambda, \Lambda^{\prime}} \mathcal{R}_{n l}(r) Y_{l \Lambda}^{*}(\theta, \phi) \eta(\mathbf{r})\left[C_{l \Lambda, \frac{1}{2} \frac{1}{2}}^{j m} C_{l^{\prime} \Lambda^{\prime}, \frac{1}{2} \frac{1}{2}}^{j^{\prime} m^{\prime}} \frac{\hbar}{2} \hat{l}_{0}(\mathbf{r}) \delta_{s \frac{1}{2}} \delta_{s^{\prime} \frac{1}{2}}\right. \\
+C_{l \Lambda, \frac{1}{2} \frac{1}{2}}^{j m} C_{l^{\prime} \Lambda^{\prime}, \frac{1}{2}-\frac{1}{2}}^{j^{\prime} m^{\prime}} \frac{\hbar}{\sqrt{2}} \hat{l}_{-1}(\mathbf{r}) \delta_{s \frac{1}{2}} \delta_{s^{\prime}-\frac{1}{2}}-C_{l \Lambda, \frac{1}{2}-\frac{1}{2}}^{j m} C_{l^{\prime} \Lambda^{\prime}, \frac{1}{2} \frac{1}{2} \frac{\hbar}{2}}^{j^{\prime} m^{\prime}} \hat{l}_{1}(\mathbf{r}) \delta_{s-\frac{1}{2}} \delta_{s^{\prime} \frac{1}{2}} \\
\left.-C_{l \Lambda, \frac{1}{2}-\frac{1}{2}}^{j m} C_{l^{\prime} \Lambda^{\prime}, \frac{1}{2}-\frac{1}{2}}^{j^{\prime} m^{\prime}} \frac{\hbar}{2} \hat{l}_{0}(\mathbf{r}) \delta_{s-\frac{1}{2}} \delta_{s^{\prime}-\frac{1}{2}}\right] \mathcal{R}_{n^{\prime} l^{\prime}}(r) Y_{l^{\prime} \Lambda^{\prime}}(\theta, \phi) \\
=-\sum_{s, s^{\prime}} \int d^{3} r \sum_{\Lambda} \mathcal{R}_{n l}(r) Y_{l \Lambda}^{*}(\theta, \phi) C_{l \Lambda, \frac{1}{2} s}^{j m} \eta(\mathbf{r})\left[\frac{\hbar}{2} \hat{l}_{0}(\mathbf{r}) \delta_{s \frac{1}{2}} \delta_{s^{\prime} \frac{1}{2}}+\frac{\hbar}{\sqrt{2}} \hat{l}_{-1}(\mathbf{r}) \delta_{s^{\frac{1}{2}}} \delta_{s^{\prime}-\frac{1}{2}}\right. \\
\left.-\frac{\hbar}{\sqrt{2}} \hat{l}_{1}(\mathbf{r}) \delta_{s-\frac{1}{2}} \delta_{s^{\prime} \frac{1}{2}}-\frac{\hbar}{2} \hat{l}_{0}(\mathbf{r}) \delta_{s-\frac{1}{2}} \delta_{s^{\prime}-\frac{1}{2}}\right] \sum_{\Lambda^{\prime}} \mathcal{R}_{n^{\prime} l^{\prime}}(r) Y_{l^{\prime} \Lambda^{\prime}}(\theta, \phi) C_{l^{\prime} \Lambda^{\prime}, \frac{1}{2} s^{\prime}}^{j^{\prime} m^{\prime}} \\
=-\sum_{s, s^{\prime}} \int d^{3} r \phi_{n l j m}^{*}(\mathbf{r}, s) \eta(\mathbf{r})\left[\frac{\hbar}{2} \hat{l}_{0}(\mathbf{r}) \delta_{s \frac{1}{2}} \delta_{s^{\prime} \frac{1}{2}}+\frac{\hbar}{\sqrt{2}} \hat{l}_{-1}(\mathbf{r}) \delta_{s \frac{1}{2}} \delta_{s^{\prime}-\frac{1}{2}}\right. \\
\left.-\frac{\hbar}{\sqrt{2}} \hat{l}_{1}(\mathbf{r}) \delta_{s-\frac{1}{2}} \delta_{s^{\prime} \frac{1}{2}}-\frac{\hbar}{2} \hat{l}_{0}(\mathbf{r}) \delta_{s-\frac{1}{2}} \delta_{s^{\prime}-\frac{1}{2}}\right] \phi_{n^{\prime} l^{\prime} j^{\prime} m^{\prime}}\left(\mathbf{r}, s^{\prime}\right) \\
=<\nu\left|\hat{h}^{\uparrow \uparrow}\right| \nu^{\prime}>+<\nu\left|\hat{h}^{\uparrow \downarrow}\right| \nu^{\prime}>+<\nu\left|h^{\downarrow \uparrow}\right| \nu^{\prime}>+<\nu\left|\hat{h}^{\downarrow \downarrow}\right| \nu^{\prime}>,
\end{gathered}
$$

where

$$
\begin{gathered}
\hat{h}^{\uparrow \uparrow}\left(\mathbf{r}, s, s^{\prime}\right)=-\frac{\hbar}{2} \eta(\mathbf{r}) \hat{l}_{0}(\mathbf{r}) \delta_{s \frac{1}{2}} \delta_{s^{\prime} \frac{1}{2}}, \quad \hat{h}^{\uparrow \downarrow}\left(\mathbf{r}, s, s^{\prime}\right)=-\frac{\hbar}{\sqrt{2}} \eta(\mathbf{r}) \hat{l}_{-1}(\mathbf{r}) \delta_{s \frac{1}{2}} \delta_{s^{\prime}-\frac{1}{2}}, \\
\hat{h}^{\downarrow \uparrow}\left(\mathbf{r}, s, s^{\prime}\right)=\frac{\hbar}{\sqrt{2}} \eta(\mathbf{r}) \hat{l}_{1}(\mathbf{r}) \delta_{s-\frac{1}{2}} \delta_{s^{\prime} \frac{1}{2}}, \quad \hat{h}^{\downarrow \downarrow}\left(\mathbf{r}, s, s^{\prime}\right)=\frac{\hbar}{2} \eta(\mathbf{r}) \hat{l}_{0}(\mathbf{r}) \delta_{s-\frac{1}{2}} \delta_{s^{\prime}-\frac{1}{2}} .
\end{gathered}
$$

Matrix elements of $\hat{h}_{l s}$ in coordinate space are derived using the completeness of the set of functions $\phi_{n l j m}(\mathbf{r}, s)$. For example

$$
\begin{array}{r}
<\mathbf{r}_{1}, s_{1}\left|\hat{h}^{\uparrow \uparrow}\right| \mathbf{r}_{2}, s_{2}>=\sum_{\nu, \nu^{\prime}}<\mathbf{r}_{1}, s_{1}|\nu><\nu| \hat{h}^{\uparrow \uparrow}\left|\nu^{\prime}><\nu^{\prime}\right| \mathbf{r}_{2}, s_{2}> \\
=-\frac{\hbar}{2} \sum_{n l j m} \sum_{n^{\prime} l^{\prime} j^{\prime} m^{\prime}} \phi_{n l j m}\left(\mathbf{r}_{1}, s_{1}\right) \sum_{s, s^{\prime}} \int d^{3} r \phi_{n l j m}^{*}(\mathbf{r}, s) \eta(\mathbf{r}) \hat{l}_{0}(\mathbf{r}) \delta_{s \uparrow} \delta_{s^{\prime} \uparrow} \phi_{n^{\prime} l^{\prime} j^{\prime} m^{\prime}}\left(\mathbf{r}, s^{\prime}\right) \phi_{n^{\prime} l^{\prime} j^{\prime} m^{\prime}}^{*}\left(\mathbf{r}_{2}, s_{2}\right) \\
=-\frac{\hbar}{2} \sum_{s, s^{\prime}} \int d^{3} r \delta\left(\mathbf{r}-\mathbf{r}_{1}\right) \delta_{s_{1} s} \eta(\mathbf{r}) \hat{l}_{0}(\mathbf{r}) \delta_{s \uparrow} \delta_{s^{\prime} \uparrow} \delta\left(\mathbf{r}-\mathbf{r}_{2}\right) \delta_{s_{2} s^{\prime}}=-\frac{\hbar}{2} \eta\left(\mathbf{r}_{1}\right) \hat{l}_{0}\left(\mathbf{r}_{1}\right) \delta\left(\mathbf{r}_{1}-\mathbf{r}_{2}\right) \delta_{s_{1} \uparrow} \delta_{s_{2} \uparrow} \\
=\frac{\hbar}{2} \hat{l}_{0}\left(\mathbf{r}_{2}\right)\left[\eta\left(\mathbf{r}_{2}\right) \delta\left(\mathbf{r}_{2}-\mathbf{r}_{1}\right)\right] \delta_{s_{1} \uparrow} \delta_{s_{2} \uparrow}=h_{l_{0}}\left(\mathbf{r}_{1}, \mathbf{r}_{2}\right) \delta_{s_{1} \uparrow} \delta_{s_{2} \uparrow},
\end{array}
$$


where

$$
h_{l_{0}}\left(\mathbf{r}_{1}, \mathbf{r}_{2}\right)=-\frac{\hbar}{2} \eta\left(\mathbf{r}_{1}\right) \hat{l}_{0}\left(\mathbf{r}_{1}\right) \delta\left(\mathbf{r}_{1}-\mathbf{r}_{2}\right)=\frac{\hbar}{2} \hat{l}_{0}\left(\mathbf{r}_{2}\right)\left[\eta\left(\mathbf{r}_{2}\right) \delta\left(\mathbf{r}_{2}-\mathbf{r}_{1}\right)\right] .
$$

Analogously we find

$$
\begin{array}{r}
<\mathbf{r}_{1}, s_{1}\left|\hat{h}_{s s^{\prime}}^{\downarrow \downarrow}\right| \mathbf{r}_{2}, s_{2}>=-h_{l_{0}}\left(\mathbf{r}_{1}, \mathbf{r}_{2}\right) \delta_{s_{1} \downarrow} \delta_{s_{2} \downarrow} \\
<\mathbf{r}_{1}, s_{1}\left|\hat{h}_{s s^{\prime}}^{\uparrow \downarrow}\right| \mathbf{r}_{2}, s_{2}>=h_{l_{-1}}\left(\mathbf{r}_{1}, \mathbf{r}_{2}\right) \delta_{s_{1} \uparrow} \delta_{s_{2} \downarrow} \\
<\mathbf{r}_{1}, s_{1}\left|\hat{h}^{\downarrow \uparrow}\right| \mathbf{r}_{2}, s_{2}>=h_{l_{1}}\left(\mathbf{r}_{1}, \mathbf{r}_{2}\right) \delta_{s_{1} \downarrow} \delta_{s_{2} \uparrow}
\end{array}
$$

with

$$
h_{l_{1}}\left(\mathbf{r}_{1}, \mathbf{r}_{2}\right)=\frac{\hbar}{\sqrt{2}} \eta\left(\mathbf{r}_{1}\right) \hat{l}_{1}\left(\mathbf{r}_{1}\right) \delta\left(\mathbf{r}_{1}-\mathbf{r}_{2}\right), \quad h_{l_{-1}}\left(\mathbf{r}_{1}, \mathbf{r}_{2}\right)=-\frac{\hbar}{\sqrt{2}} \eta\left(\mathbf{r}_{1}\right) \hat{l}_{-1}\left(\mathbf{r}_{1}\right) \delta\left(\mathbf{r}_{1}-\mathbf{r}_{2}\right) .
$$

As a result

$$
<\mathbf{r}_{1}, s_{1}\left|\hat{h}_{l s}\right| \mathbf{r}_{2}, s_{2}>=h_{l_{0}}\left(\mathbf{r}_{1}, \mathbf{r}_{2}\right)\left[\delta_{s_{1} \uparrow} \delta_{s_{2} \uparrow}-\delta_{s_{1} \downarrow} \delta_{s_{2} \downarrow}\right]+h_{l_{-1}}\left(\mathbf{r}_{1}, \mathbf{r}_{2}\right) \delta_{s_{1} \uparrow} \delta_{s_{2} \downarrow}+h_{l_{1}}\left(\mathbf{r}_{1}, \mathbf{r}_{2}\right) \delta_{s_{1} \downarrow} \delta_{s_{2} \uparrow} .
$$

According to (52)-(54) its Wigner transformation reads:

$$
h_{l s}(\mathbf{r}, \mathbf{p})=-\frac{\hbar}{2} \eta(\mathbf{r})\left\{l_{0}(\mathbf{r}, \mathbf{p})\left[\delta_{s_{1} \uparrow} \delta_{s_{2} \uparrow}-\delta_{s_{1} \downarrow} \delta_{s_{2} \downarrow}\right]+\sqrt{2} l_{-1}(\mathbf{r}, \mathbf{p}) \delta_{s_{1} \uparrow} \delta_{s_{2} \downarrow}-\sqrt{2} l_{1}(\mathbf{r}, \mathbf{p}) \delta_{s_{1} \downarrow} \delta_{s_{2} \uparrow}\right\} .
$$

Taking into account equations (19) and (21) one can show that second and third equations of (3) are complex conjugated if the following relation is fulfilled:

$$
<\mathbf{r}|\hat{\rho}| \mathbf{r}^{\prime \prime}>^{\uparrow \downarrow}=<\mathbf{r}^{\prime \prime}|\hat{\rho}| \mathbf{r}>^{\downarrow \uparrow *},
$$

i.e. if the density matrix is Hermitian as it must be.

\section{Equations of motion}

Integrating the set of equations (5) over the phase space with the weights

$$
W=\{r \otimes p\}_{\lambda \mu},\{r \otimes r\}_{\lambda \mu},\{p \otimes p\}_{\lambda \mu} \text { and } 1
$$

one gets the dynamical equations for the following collective variables:

$$
L_{\lambda \mu}^{ \pm}(t)=\int d(\mathbf{p}, \mathbf{r})\{r \otimes p\}_{\lambda \mu} f^{ \pm}(\mathbf{r}, \mathbf{p}, t),
$$




$$
\begin{aligned}
& L_{\lambda \mu}^{u}(t)=\int d(\mathbf{p}, \mathbf{r})\{r \otimes p\}_{\lambda \mu} f^{u}(\mathbf{r}, \mathbf{p}, t), \\
& L_{\lambda \mu}^{d}(t)=\int d(\mathbf{p}, \mathbf{r})\{r \otimes p\}_{\lambda \mu} f^{d}(\mathbf{r}, \mathbf{p}, t), \\
& R_{\lambda \mu}^{ \pm}(t)= \int d(\mathbf{p}, \mathbf{r})\{r \otimes r\}_{\lambda \mu} f^{ \pm}(\mathbf{r}, \mathbf{p}, t), \\
& R_{\lambda \mu}^{u}(t)= \int d(\mathbf{p}, \mathbf{r})\{r \otimes r\}_{\lambda \mu} f^{u}(\mathbf{r}, \mathbf{p}, t), \\
& R_{\lambda \mu}^{d}(t)= \int d(\mathbf{p}, \mathbf{r})\{r \otimes r\}_{\lambda \mu} f^{d}(\mathbf{r}, \mathbf{p}, t), \\
& P_{\lambda \mu}^{ \pm}(t)= \int d(\mathbf{p}, \mathbf{r})\{p \otimes p\}_{\lambda \mu} f^{ \pm}(\mathbf{r}, \mathbf{p}, t), \\
& P_{\lambda \mu}^{u}(t)= \int d(\mathbf{p}, \mathbf{r})\{p \otimes p\}_{\lambda \mu} f^{u}(\mathbf{r}, \mathbf{p}, t), \\
& P_{\lambda \mu}^{d}(t)= \int d(\mathbf{p}, \mathbf{r})\{p \otimes p\}_{\lambda \mu} f^{d}(\mathbf{r}, \mathbf{p}, t), \\
& F^{ \pm}(t)=\int d(\mathbf{p}, \mathbf{r}) f^{ \pm}(\mathbf{r}, \mathbf{p}, t), \\
& F^{u}(t)=\int d(\mathbf{p}, \mathbf{r}) f^{u}(\mathbf{r}, \mathbf{p}, t), \\
& F^{d}(t)=\int d(\mathbf{p}, \mathbf{r}) f^{d}(\mathbf{r}, \mathbf{p}, t) .
\end{aligned}
$$

We already named functions $f^{+}(\mathbf{r}, \mathbf{p}, t)$ and $f^{-}(\mathbf{r}, \mathbf{p}, t)$ as spin-scalar and spin-vector ones. So it will be natural to name the respective collective variables $X_{\lambda \mu}^{+}(t)$ and $X_{\lambda \mu}^{-}(t)$ as spin-scalar and spin-vector variables. The required expressions for $h^{ \pm}, h^{\uparrow \downarrow}$ and $h^{\downarrow \uparrow}$ are

$$
h^{+}=m \omega^{2} r^{2}+2 \sum_{\mu}(-1)^{\mu} Z_{2 \mu}^{\tau+}\{r \otimes r\}_{2-\mu}, \quad h^{-}=-\hbar \eta l_{0}, h^{\uparrow \downarrow}=-\frac{\hbar}{\sqrt{2}} \eta l_{-1}, h^{\downarrow \uparrow}=\frac{\hbar}{\sqrt{2}} \eta l_{1} .
$$

The integration yields:

$$
\begin{aligned}
\dot{L}_{\lambda \mu}^{+}= & \frac{1}{m} P_{\lambda \mu}^{+}-m \omega^{2} R_{\lambda \mu}^{+}+2 \sqrt{5} \sum_{j=0}^{2} \sqrt{2 j+1}\left\{\begin{array}{l}
11 j \\
2 \lambda 1
\end{array}\right\}\left\{Z_{2}^{+} \otimes R_{j}^{+}\right\}_{\lambda \mu} \\
& -i \hbar \frac{\eta}{2}\left[\mu L_{\lambda \mu}^{-}+\sqrt{(\lambda-\mu)(\lambda+\mu+1)} L_{\lambda \mu+1}^{u}+\sqrt{(\lambda+\mu)(\lambda-\mu+1)} L_{\lambda \mu-1}^{d}\right], \\
\dot{L}_{\lambda \mu}^{-}= & \frac{1}{m} P_{\lambda \mu}^{-}-m \omega^{2} R_{\lambda \mu}^{-}+2 \sqrt{5} \sum_{j=0}^{2} \sqrt{2 j+1}\left\{\begin{array}{l}
11 j \\
2 \lambda 1
\end{array}\right\}\left\{Z_{2}^{+} \otimes R_{j}^{-}\right\}_{\lambda \mu} \\
& +i \eta \sqrt{2} \int d(\mathbf{p}, \mathbf{r})(r p)_{\lambda \mu}\left[l_{1} f^{u}+l_{-1} f^{d}\right] \\
& -i \hbar \frac{\eta}{2} \mu L_{\lambda \mu}^{+}-\frac{\hbar^{2}}{2} \eta \delta_{\lambda, 1}\left[\delta_{\mu,-1} F^{u}+\delta_{\mu, 1} F^{d}\right], \\
\dot{L}_{\lambda \mu+1}^{u}= & \frac{1}{m} P_{\lambda \mu+1}^{u}-m \omega^{2} R_{\lambda \mu+1}^{u}+2 \sqrt{5} \sum_{j=0}^{2} \sqrt{2 j}+1 \\
& \left.-i \frac{\eta}{\sqrt{2}} \int d(\mathbf{p}, \mathbf{r})(r p)_{\lambda \mu+1}^{11 j}{ }_{2 \lambda 1}\right\}\left\{Z_{-1}^{+} f^{-}-\sqrt{2} l_{0} f^{u}\right]
\end{aligned}
$$




$$
\begin{aligned}
& -i \hbar \frac{\eta}{4} \sqrt{(\lambda-\mu)(\lambda+\mu+1)} L_{\lambda \mu}^{+}+\frac{\hbar^{2}}{2} \eta \delta_{\lambda, 1}\left[\delta_{\mu, 0} F^{-}+\frac{1}{\sqrt{2}} \delta_{\mu,-1} F^{u}\right], \\
& \dot{L}_{\lambda \mu-1}^{d}=\frac{1}{m} P_{\lambda \mu-1}^{d}-m \omega^{2} R_{\lambda \mu-1}^{d}+2 \sqrt{5} \sum_{j=0}^{2} \sqrt{2 j+1}\left\{\begin{array}{l}
11 j \\
2 \lambda 1
\end{array}\right\}\left\{Z_{2}^{+} \otimes R_{j}^{d}\right\}_{\lambda \mu-1} \\
& -i \frac{\eta}{\sqrt{2}} \int d(\mathbf{p}, \mathbf{r})(r p)_{\lambda \mu-1}\left[l_{1} f^{-}+\sqrt{2} l_{0} f^{d}\right] \\
& -i \hbar \frac{\eta}{4} \sqrt{(\lambda+\mu)(\lambda-\mu+1)} L_{\lambda \mu}^{+}+\frac{\hbar^{2}}{4} \eta \delta_{\lambda, 1}\left[\delta_{\mu, 0} F^{-}-\sqrt{2} \delta_{\mu, 1} F^{d}\right], \\
& \dot{F}^{-}=2 \eta\left[L_{1-1}^{d}+L_{11}^{u}\right] \text {, } \\
& \dot{F}^{u}=-\eta\left[L_{1-1}^{-}-\sqrt{2} L_{10}^{u}\right], \\
& \dot{F}^{d}=-\eta\left[L_{11}^{-}+\sqrt{2} L_{10}^{d}\right] \text {, } \\
& \dot{R}_{\lambda \mu}^{+}=\frac{2}{m} L_{\lambda \mu}^{+} \\
& -i \hbar \frac{\eta}{2}\left[\mu R_{\lambda \mu}^{-}+\sqrt{(\lambda-\mu)(\lambda+\mu+1)} R_{\lambda \mu+1}^{u}+\sqrt{(\lambda+\mu)(\lambda-\mu+1)} R_{\lambda \mu-1}^{d}\right], \\
& \dot{R}_{\lambda \mu}^{-}=\frac{2}{m} L_{\lambda \mu}^{-}-i \hbar \frac{\eta}{2} \mu R_{\lambda \mu}^{+}+i \eta \sqrt{2} \int d(\mathbf{p}, \mathbf{r})\left(r^{2}\right)_{\lambda \mu}\left[l_{1} f^{u}+l_{-1} f^{d}\right], \\
& \dot{R}_{\lambda \mu+1}^{u}=\frac{2}{m} L_{\lambda \mu+1}^{u}-i \frac{\eta}{\sqrt{2}} \int d(\mathbf{p}, \mathbf{r})\left(r^{2}\right)_{\lambda \mu+1}\left[l_{-1} f^{-}-\sqrt{2} l_{0} f^{u}\right] \\
& -i \hbar \frac{\eta}{4} \sqrt{(\lambda-\mu)(\lambda+\mu+1)} R_{\lambda \mu}^{+} \\
& \dot{R}_{\lambda \mu-1}^{d}=\frac{2}{m} L_{\lambda \mu-1}^{d}-i \frac{\eta}{\sqrt{2}} \int d(\mathbf{p}, \mathbf{r})\left(r^{2}\right)_{\lambda \mu-1}\left[l_{1} f^{-}+\sqrt{2} l_{0} f^{d}\right] \\
& -i \hbar \frac{\eta}{4} \sqrt{(\lambda+\mu)(\lambda-\mu+1)} R_{\lambda \mu}^{+} \\
& \dot{P}_{\lambda \mu}^{+}=-2 m \omega^{2} L_{\lambda \mu}^{+}+4 \sqrt{5} \sum_{j=0}^{2} \sqrt{2 j+1}\left\{\begin{array}{c}
11 j \\
2 \lambda 1
\end{array}\right\}\left\{Z_{2}^{+} \otimes L_{j}^{+}\right\}_{\lambda \mu} \\
& -i \hbar \frac{\eta}{2}\left[\mu P_{\lambda \mu}^{-}+\sqrt{(\lambda-\mu)(\lambda+\mu+1)} P_{\lambda \mu+1}^{u}+\sqrt{(\lambda+\mu)(\lambda-\mu+1)} P_{\lambda \mu-1}^{d}\right] \text {, } \\
& \dot{P}_{\lambda \mu}^{-}=-2 m \omega^{2} L_{\lambda \mu}^{-}+4 \sqrt{5} \sum_{j=0}^{2} \sqrt{2 j+1}\left\{\begin{array}{l}
11 j \\
2 \lambda 1
\end{array}\right\}\left\{Z_{2}^{+} \otimes L_{j}^{-}\right\}_{\lambda \mu} \\
& -i \hbar \frac{\eta}{2} \mu P_{\lambda \mu}^{+}+i \eta \sqrt{2} \int d(\mathbf{p}, \mathbf{r})\left(p^{2}\right)_{\lambda \mu}\left[l_{1} f^{u}+l_{-1} f^{d}\right], \\
& \dot{P}_{\lambda \mu+1}^{u}=-2 m \omega^{2} L_{\lambda \mu+1}^{u}+4 \sqrt{5} \sum_{j=0}^{2} \sqrt{2 j+1}\left\{\begin{array}{l}
11 j \\
2 \lambda 1
\end{array}\right\}\left\{Z_{2}^{+} \otimes L_{j}^{u}\right\}_{\lambda \mu+1} \\
& -i \hbar \frac{\eta}{4} \sqrt{(\lambda-\mu)(\lambda+\mu+1)} P_{\lambda \mu}^{+}-i \frac{\eta}{\sqrt{2}} \int d(\mathbf{p}, \mathbf{r})\left(p^{2}\right)_{\lambda \mu+1}\left[l_{-1} f^{-}-\sqrt{2} l_{0} f^{u}\right], \\
& \dot{P}_{\lambda \mu-1}^{d}=-2 m \omega^{2} L_{\lambda \mu-1}^{d}+4 \sqrt{5} \sum_{j=0}^{2} \sqrt{2 j+1}\left\{\begin{array}{l}
11 j \\
2 \lambda 1
\end{array}\right\}\left\{Z_{2}^{+} \otimes L_{j}^{d}\right\}_{\lambda \mu-1}
\end{aligned}
$$




$$
-i \hbar \frac{\eta}{4} \sqrt{(\lambda+\mu)(\lambda-\mu+1)} P_{\lambda \mu}^{+}-i \frac{\eta}{\sqrt{2}} \int d(\mathbf{p}, \mathbf{r})\left(p^{2}\right)_{\lambda \mu-1}\left[l_{1} f^{-}+\sqrt{2} l_{0} f^{d}\right],
$$

where $\left\{\begin{array}{l}11 j \\ 2 \lambda 1\end{array}\right\}$ is the Wigner $6 j$-symbol. For the sake of simplicity the time dependence of tensors is not written out. It is easy to see that this set of equations for moments of zero and second orders is not closed due to the integral terms of the type $\int d(\mathbf{p}, \mathbf{r})\left(p^{2}\right)_{\lambda \mu-1}\left[l_{1} f^{-}+\sqrt{2} l_{0} f^{d}\right]$ (see last equation). These terms generate moments of fourth order. They will be neglected according to the rules of the WFM method [15].

We are interested in the scissors mode with quantum number $K^{\pi}=1^{+}$. Therefore we only need the part of equations (27) with $\mu=1$. These equations are nonlinear and will be solved in small amplitude approximation.

\subsection{Linearized equations $(\mu=1)$}

Writing all variables as a sum of their equilibrium value plus a small deviation

$$
R_{\lambda \mu}(t)=R_{\lambda \mu}^{e q}+\mathcal{R}_{\lambda \mu}(t), \quad P_{\lambda \mu}(t)=P_{\lambda \mu}^{e q}+\mathcal{P}_{\lambda \mu}(t), \quad L_{\lambda \mu}(t)=L_{\lambda \mu}^{e q}+\mathcal{L}_{\lambda \mu}(t)
$$

and neglecting quadratic deviations, one obtains the desired equations. Naturally one needs to know the equilibrium values of all variables. Obvious equilibrium conditions for an axially symmetric nucleus are:

$$
R_{2 \pm 1}^{+}(e q)=R_{2 \pm 2}^{+}(e q)=0, \quad R_{20}^{+}(e q) \neq 0 .
$$

It is obvious that all ground state properties of the system of spin up nucleons are identical to that of the system of nucleons with spin down. Therefore

$$
R_{\lambda \mu}^{-}(e q)=P_{\lambda \mu}^{-}(e q)=L_{\lambda \mu}^{-}(e q)=0 .
$$

We also will suppose

$$
L_{\lambda \mu}^{+}(e q)=L_{\lambda \mu}^{u}(e q)=L_{\lambda \mu}^{d}(e q)=0 \quad \text { and } \quad R_{\lambda \mu}^{u}(e q)=R_{\lambda \mu}^{d}(e q)=0 .
$$

With the help of the above equilibrium relations one arrives at the following set of equations:

$$
\dot{\mathcal{L}}_{21}^{+}=\frac{1}{m} \mathcal{P}_{21}^{+}-m \omega^{2} \mathcal{R}_{21}^{+}+\frac{2}{\sqrt{3}} R_{00}^{+}(e q) \mathcal{Z}_{21}^{+}-\frac{1}{\sqrt{6}}\left[R_{20}^{+}(e q) \mathcal{Z}_{21}^{+}+Z_{20}^{+}(e q) \mathcal{R}_{21}^{+}\right]
$$




$$
\begin{aligned}
& -i \hbar \frac{\eta}{2}\left[\mathcal{L}_{21}^{-}+2 \mathcal{L}_{22}^{u}+2 \sqrt{\frac{3}{2}} \mathcal{L}_{20}^{d}\right] \\
& \dot{\mathcal{L}}_{21}^{-}=\frac{1}{m} \mathcal{P}_{21}^{-}-m \omega^{2} \mathcal{R}_{21}^{-}-\frac{1}{\sqrt{6}} Z_{20}^{+}(e q) \mathcal{R}_{21}^{-}-i \hbar \frac{\eta}{2} \mathcal{L}_{21}^{+}, \\
& \dot{\mathcal{L}}_{22}^{u}=\frac{1}{m} \mathcal{P}_{22}^{u}-m \omega^{2} \mathcal{R}_{22}^{u}-\sqrt{\frac{2}{3}} Z_{20}^{+}(e q) \mathcal{R}_{22}^{u}-i \hbar \frac{\eta}{2} \mathcal{L}_{21}^{+}, \\
& \dot{\mathcal{L}}_{20}^{d}=\frac{1}{m} \mathcal{P}_{20}^{d}-m \omega^{2} \mathcal{R}_{20}^{d}-\sqrt{\frac{2}{3}} Z_{20}^{+}(e q) \mathcal{R}_{20}^{d}+\frac{2}{\sqrt{3}} Z_{20}^{+}(e q) \mathcal{R}_{00}^{d}-i \hbar \frac{\eta}{2} \sqrt{\frac{3}{2}} \mathcal{L}_{21}^{+}, \\
& \dot{\mathcal{L}}_{11}^{+}=\sqrt{\frac{3}{2}}\left[R_{20}^{+}(e q) \mathcal{Z}_{21}^{+}-Z_{20}^{+}(e q) \mathcal{R}_{21}^{+}\right]-i \hbar \frac{\eta}{2}\left[\mathcal{L}_{11}^{-}+\sqrt{2} \mathcal{L}_{10}^{d}\right], \\
& \dot{\mathcal{L}}_{11}^{-}=-\sqrt{\frac{3}{2}} Z_{20}^{+}(e q) \mathcal{R}_{21}^{-}-i \hbar \frac{\eta}{2} \mathcal{L}_{11}^{+}-\frac{\hbar^{2}}{2} \eta F^{d} \\
& \dot{\mathcal{L}}_{10}^{d}=-i \hbar \frac{\eta}{2 \sqrt{2}} \mathcal{L}_{11}^{+}-\frac{\hbar^{2}}{4} \eta \sqrt{2} F^{d}, \\
& \dot{F}^{d}=-\eta\left[\mathcal{L}_{11}^{-}+\sqrt{2} \mathcal{L}_{10}^{d}\right], \\
& \dot{\mathcal{R}}_{21}^{+}=\frac{2}{m} \mathcal{L}_{21}^{+}-i \hbar \frac{\eta}{2}\left[\mathcal{R}_{21}^{-}+2 \mathcal{R}_{22}^{u}+2 \sqrt{\frac{3}{2}} \mathcal{R}_{20}^{d}\right], \\
& \dot{\mathcal{R}}_{21}^{-}=\frac{2}{m} \mathcal{L}_{21}^{-}-i \hbar \frac{\eta}{2} \mathcal{R}_{21}^{+}, \\
& \dot{\mathcal{R}}_{22}^{u}=\frac{2}{m} \mathcal{L}_{22}^{u}-i \hbar \frac{\eta}{2} \mathcal{R}_{21}^{+}, \\
& \dot{\mathcal{R}}_{20}^{d}=\frac{2}{m} \mathcal{L}_{20}^{d}-i \hbar \frac{\eta}{2} \sqrt{\frac{3}{2}} \mathcal{R}_{21}^{+}, \\
& \dot{\mathcal{P}}_{21}^{+}=-2 m \omega^{2} \mathcal{L}_{21}^{+}+\sqrt{6} Z_{20}^{+}(e q) \mathcal{L}_{11}^{+}-\sqrt{\frac{2}{3}} Z_{20}^{+}(e q) \mathcal{L}_{21}^{+} \\
& -i \hbar \frac{\eta}{2}\left[\mathcal{P}_{21}^{-}+2 \mathcal{P}_{22}^{u}+2 \sqrt{\frac{3}{2}} \mathcal{P}_{20}^{d}\right] \\
& \dot{\mathcal{P}}_{21}^{-}=-2 m \omega^{2} \mathcal{L}_{21}^{-}+\sqrt{6} Z_{20}^{+}(e q) \mathcal{L}_{11}^{-}-\sqrt{\frac{2}{3}} Z_{20}^{+}(e q) \mathcal{L}_{21}^{-}-i \hbar \frac{\eta}{2} \mathcal{P}_{21}^{+}, \\
& \dot{\mathcal{P}}_{22}^{u}=-2 m \omega^{2} \mathcal{L}_{22}^{u}+2 \sqrt{\frac{2}{3}} Z_{20}^{+}(e q) \mathcal{L}_{22}^{u}-i \hbar \frac{\eta}{2} \mathcal{P}_{21}^{+}, \\
& \dot{\mathcal{P}}_{20}^{d}=-2 m \omega^{2} \mathcal{L}_{20}^{d}-2 \sqrt{\frac{2}{3}} Z_{20}^{+}(e q) \mathcal{L}_{20}^{d}+2 \sqrt{\frac{4}{3}} Z_{20}^{+}(e q) \mathcal{L}_{00}^{d}-i \hbar \frac{\eta}{2} \sqrt{\frac{3}{2}} \mathcal{P}_{21}^{+}, \\
& \dot{\mathcal{L}}_{00}^{d}=\frac{1}{m} \mathcal{P}_{00}^{d}-m \omega^{2} \mathcal{R}_{00}^{d}+\frac{2}{\sqrt{3}} Z_{20}^{+}(e q) \mathcal{R}_{20}^{d}, \\
& \dot{\mathcal{R}}_{00}^{d}=\frac{2}{m} \mathcal{L}_{00}^{d} \\
& \dot{\mathcal{P}}_{00}^{d}=-2 m \omega^{2} \mathcal{L}_{00}^{d}+2 \sqrt{\frac{4}{3}} Z_{20}^{+}(e q) \mathcal{L}_{20}^{d} .
\end{aligned}
$$


Let us recall that all variables and equilibrium quantities $R_{20}^{+}(e q)$ and $Z_{20}^{+}(e q)$ in (31) have isospin indices $\tau=\mathrm{n}$, p. All the difference between neutron and proton systems lies in the mean field quantity $Z_{20}^{\tau+}(e q)$, which is different for neutrons and protons (see (8)).

\subsection{Isovector, isoscalar}

It is convenient to rewrite the equations (31) in terms of isovector and isoscalar variables

$$
\begin{aligned}
& R_{\lambda \mu}=R_{\lambda \mu}^{\mathrm{n}}+R_{\lambda \mu}^{\mathrm{p}}, \quad P_{\lambda \mu}=P_{\lambda \mu}^{\mathrm{n}}+P_{\lambda \mu}^{\mathrm{p}}, \quad L_{\lambda \mu}=L_{\lambda \mu}^{\mathrm{n}}+L_{\lambda \mu}^{\mathrm{p}}, \\
& \bar{R}_{\lambda \mu}=R_{\lambda \mu}^{\mathrm{n}}-R_{\lambda \mu}^{\mathrm{p}}, \quad \bar{P}_{\lambda \mu}=P_{\lambda \mu}^{\mathrm{n}}-P_{\lambda \mu}^{\mathrm{p}}, \quad \bar{L}_{\lambda \mu}=L_{\lambda \mu}^{\mathrm{n}}-L_{\lambda \mu}^{\mathrm{p}} .
\end{aligned}
$$

It is natural to define also isovector and isoscalar strength constants $\chi_{1}=\frac{1}{2}(\chi-\bar{\chi})$ and $\chi_{0}=$ $\frac{1}{2}(\chi+\bar{\chi})$ connected by the relation $\chi_{1}=\alpha \chi_{0}[16]$. Then the equations for the neutron and proton systems are transformed into isovector and isoscalar ones. The equations for the isovector system are given by

$$
\begin{aligned}
& \dot{\overline{\mathcal{L}}}_{21}^{+}=\frac{1}{m} \overline{\mathcal{P}}_{21}^{+}-\left[m \omega^{2}-\frac{2}{\sqrt{3}} \alpha \chi_{0} R_{00}^{+}(e q)+\frac{1}{\sqrt{6}}(1+\alpha) \chi_{0} R_{20}^{+}(e q)\right] \overline{\mathcal{R}}_{21}^{+} \\
& -i \hbar \frac{\eta}{2}\left[\overline{\mathcal{L}}_{21}^{-}+2 \overline{\mathcal{L}}_{22}^{u}+2 \sqrt{\frac{3}{2}} \overline{\mathcal{L}}_{20}^{d}\right] \\
& \dot{\overline{\mathcal{L}}}_{21}^{-}=\frac{1}{m} \overline{\mathcal{P}}_{21}^{-}-\left[m \omega^{2}+\frac{1}{\sqrt{6}} \chi_{0} R_{20}(e q)\right] \overline{\mathcal{R}}_{21}^{-}-i \hbar \frac{\eta}{2} \overline{\mathcal{L}}_{21}^{+} \text {, } \\
& \dot{\overline{\mathcal{L}}}_{22}^{u}=\frac{1}{m} \overline{\mathcal{P}}_{22}^{u}-\left[m \omega^{2}-\sqrt{\frac{2}{3}} \chi_{0} R_{20}(e q)\right] \overline{\mathcal{R}}_{22}^{u}-i \hbar \frac{\eta}{2} \overline{\mathcal{L}}_{21}^{+}, \\
& \dot{\overline{\mathcal{L}}}_{20}^{d}=\frac{1}{m} \overline{\mathcal{P}}_{20}^{d}-\left[m \omega^{2}+\sqrt{\frac{2}{3}} \chi_{0} R_{20}(e q)\right] \overline{\mathcal{R}}_{20}^{d}+\frac{2}{\sqrt{3}} \chi_{0} R_{20}(e q) \overline{\mathcal{R}}_{00}^{d}-i \hbar \frac{\eta}{2} \sqrt{\frac{3}{2}} \overline{\mathcal{L}}_{21}^{+}, \\
& \dot{\overline{\mathcal{L}}}_{11}^{+}=-\sqrt{\frac{3}{2}}(1-\alpha) \chi_{0} R_{20}(e q) \overline{\mathcal{R}}_{21}^{+}-i \hbar \frac{\eta}{2}\left[\overline{\mathcal{L}}_{11}^{-}+\sqrt{2} \overline{\mathcal{L}}_{10}^{d}\right] \\
& \dot{\overline{\mathcal{L}}}_{11}^{-}=-\sqrt{\frac{3}{2}} \chi_{0} R_{20}(e q) \overline{\mathcal{R}}_{21}^{-}-\hbar \frac{\eta}{2}\left[i \overline{\mathcal{L}}_{11}^{+}+\hbar \bar{F}^{d}\right] \\
& \dot{\overline{\mathcal{L}}}_{10}^{d}=-\hbar \frac{\eta}{2 \sqrt{2}}\left[i \overline{\mathcal{L}}_{11}^{+}+\hbar \bar{F}^{d}\right] \text {, } \\
& \dot{\bar{F}}^{d}=-\eta\left[\overline{\mathcal{L}}_{11}^{-}+\sqrt{2} \overline{\mathcal{L}}_{10}^{d}\right] \text {, } \\
& \dot{\overline{\mathcal{R}}}_{21}^{+}=\frac{2}{m} \overline{\mathcal{L}}_{21}^{+}-i \hbar \frac{\eta}{2}\left[\overline{\mathcal{R}}_{21}^{-}+2 \overline{\mathcal{R}}_{22}^{u}+2 \sqrt{\frac{3}{2}} \overline{\mathcal{R}}_{20}^{d}\right] \text {, } \\
& \dot{\overline{\mathcal{R}}}_{21}^{-}=\frac{2}{m} \overline{\mathcal{L}}_{21}^{-}-i \hbar \frac{\eta}{2} \overline{\mathcal{R}}_{21}^{+},
\end{aligned}
$$




$$
\begin{aligned}
& \dot{\overline{\mathcal{R}}}_{22}^{u}=\frac{2}{m} \overline{\mathcal{L}}_{22}^{u}-i \hbar \frac{\eta}{2} \overline{\mathcal{R}}_{21}^{+} \\
& \dot{\overline{\mathcal{R}}}_{20}^{d}=\frac{2}{m} \overline{\mathcal{L}}_{20}^{d}-i \hbar \frac{\eta}{2} \sqrt{\frac{3}{2}} \overline{\mathcal{R}}_{21}^{+} \\
& \dot{\overline{\mathcal{P}}}_{21}^{+}=-\left[2 m \omega^{2}+\sqrt{\frac{2}{3}} \chi_{0} R_{20}(e q)\right] \overline{\mathcal{L}}_{21}^{+}+\sqrt{6} \chi_{0} R_{20}(e q) \overline{\mathcal{L}}_{11}^{+}-i \hbar \frac{\eta}{2}\left[\overline{\mathcal{P}}_{21}^{-}+2 \overline{\mathcal{P}}_{22}^{u}+2 \sqrt{\frac{3}{2}} \overline{\mathcal{P}}_{20}^{d}\right] \\
& \dot{\overline{\mathcal{P}}}_{21}^{-}=-\left[2 m \omega^{2}+\sqrt{\frac{2}{3}} \chi_{0} R_{20}(e q)\right] \overline{\mathcal{L}}_{21}^{-}+\sqrt{6} \chi_{0} R_{20}(e q) \overline{\mathcal{L}}_{11}^{-}-i \hbar \frac{\eta}{2} \overline{\mathcal{P}}_{21}^{+} \\
& \dot{\overline{\mathcal{P}}}_{22}^{u}=-\left[2 m \omega^{2}-2 \sqrt{\frac{2}{3}} \chi_{0} R_{20}(e q)\right] \overline{\mathcal{L}}_{22}^{u}-i \hbar \frac{\eta}{2} \overline{\mathcal{P}}_{21}^{+}, \\
& \dot{\overline{\mathcal{P}}}_{20}^{d}=-\left[2 m \omega^{2}+2 \sqrt{\frac{2}{3}} \chi_{0} R_{20}(e q)\right] \overline{\mathcal{L}}_{20}^{d}+\frac{4}{\sqrt{3}} \chi_{0} R_{20}(e q) \overline{\mathcal{L}}_{00}^{d}-i \hbar \frac{\eta}{2} \sqrt{\frac{3}{2}} \overline{\mathcal{P}}_{21}^{+} \\
& \dot{\overline{\mathcal{L}}}_{00}^{d}=\frac{1}{m} \overline{\mathcal{P}}_{00}^{d}-m \omega^{2} \overline{\mathcal{R}}_{00}^{d}+\frac{2}{\sqrt{3}} \chi_{0} R_{20}(e q) \overline{\mathcal{R}}_{20}^{d}, \\
& \dot{\overline{\mathcal{R}}}_{00}^{d}=\frac{2}{m} \overline{\mathcal{L}}_{00}^{d}, \\
& \dot{\overline{\mathcal{P}}}_{00}^{d}=-2 m \omega^{2} \overline{\mathcal{L}}_{00}^{d}+\frac{4}{\sqrt{3}} \chi_{0} R_{20}(e q) \overline{\mathcal{L}}_{20}^{d} .
\end{aligned}
$$

The isoscalar set of equations is easily obtained from (32) by taking $\alpha=1$.

\subsection{Angular momentum conservation}

The set of equations (32) contains three integrals of motion (see Appendix B). The first one is (in the isoscalar case) the conservation of total angular momentum $\left.\left.\left.<\hat{J}_{1}\right\rangle=<\hat{l}_{1}\right\rangle+<\hat{S}_{1}\right\rangle$. By definition

$$
\begin{array}{r}
<\hat{l}_{1}>=\operatorname{Tr}\left(\hat{l}_{1} \hat{\rho}\right)=\sum_{s} \int d^{3} r \int d^{3} r^{\prime}<\mathbf{r}\left|\hat{l}_{1}\right| \mathbf{r}^{\prime}><\mathbf{r}^{\prime}, s|\hat{\rho}| \mathbf{r}, s> \\
=\sum_{s} \int d^{3} r \int d^{3} r^{\prime} \hat{l}_{1}(\mathbf{r}) \delta\left(\mathbf{r}-\mathbf{r}^{\prime}\right)<\mathbf{r}^{\prime}, s|\hat{\rho}| \mathbf{r}, s>=\int d^{3} r \hat{l}_{1}(\mathbf{r})\left[<\mathbf{r}|\hat{\rho}| \mathbf{r}>^{\uparrow \uparrow}+<\mathbf{r}|\hat{\rho}| \mathbf{r}>^{\downarrow \downarrow}\right] \\
=\int d(\mathbf{p r}) l_{1}(\mathbf{r}, \mathbf{p}) f^{+}(\mathbf{r}, \mathbf{p})=-i \sqrt{2} \int d(\mathbf{p r})\{r \otimes p\}_{11} f^{+}(\mathbf{r}, \mathbf{p})=-i \sqrt{2} L_{11}^{+} .
\end{array}
$$

The average value of the spin operator $\hat{S}_{1}$ reads:

$$
\begin{array}{r}
<\hat{S}_{1}>=\operatorname{Tr}\left(\hat{S}_{1} \hat{\rho}\right)=\sum_{s, s^{\prime}} \int d^{3} r<s\left|\hat{S}_{1}\right| s^{\prime}><\mathbf{r}, s^{\prime}|\hat{\rho}| \mathbf{r}, s> \\
=\sum_{s, s^{\prime}}<s\left|\hat{S}_{1}\right| s^{\prime}>\int d(\mathbf{p r}) f^{s^{\prime} s}(\mathbf{r}, \mathbf{p})=-\frac{\hbar}{\sqrt{2}} \sum_{s, s^{\prime}} \delta_{s \uparrow} \delta_{s^{\prime} \downarrow} F^{s^{\prime} s}=-\frac{\hbar}{\sqrt{2}} F^{\downarrow \uparrow} \equiv-\frac{\hbar}{\sqrt{2}} F^{d}
\end{array}
$$


As a result $<\hat{J}_{1}>=-\frac{1}{\sqrt{2}}\left(2 i L_{11}^{+}+\hbar F^{d}\right)$. It is easy to see that such combination of the respective equations in $(32)$ is equal to zero in the isoscalar case $(\alpha=1)$, i.e. the total angular momentum is conserved.

\section{$5 \quad$ Energies and excitation probabilities}

Imposing the time evolution via $e^{i \Omega t}$ for all variables one transforms (32) into a set of algebraic equations. Eigenfrequencies are found as the zeros of its secular equation. Excitation probabilities are calculated with the help of the theory of linear response of the system to a weak external field

$$
\hat{O}(t)=\hat{O} \exp (-i \Omega t)+\hat{O}^{\dagger} \exp (i \Omega t)
$$

The detailed explanation can be found in [16]. Here we only will recall the main points. The matrix elements of the operator $\hat{O}$ obey the relationship

$$
\left|<\psi_{a}\right| \hat{O}\left|\psi_{0}>\right|^{2}=\hbar \lim _{\Omega \rightarrow \Omega_{a}}\left(\Omega-\Omega_{a}\right) \overline{<\psi^{\prime}|\hat{O}| \psi^{\prime}>\exp (-i \Omega t)}
$$

where $\psi_{0}$ and $\psi_{a}$ are the stationary wave functions of the unperturbed ground and excited states; $\psi^{\prime}$ is the wave function of the perturbed ground state, $\Omega_{a}=\left(E_{a}-E_{0}\right) / \hbar$ are the normal frequencies, the bar means averaging over a time interval much larger than $1 / \Omega$.

To calculate the magnetic transition probability, it is necessary to excite the system by the following external field:

$$
\hat{O}_{\lambda \mu^{\prime}}=\mu_{N}\left(g_{s} \hat{\mathbf{S}} / \hbar-i g_{l} \frac{2}{\lambda+1}[\mathbf{r} \times \nabla]\right) \nabla\left(r^{\lambda} Y_{\lambda \mu^{\prime}}\right), \quad \mu_{N}=\frac{e \hbar}{2 m c}
$$

Here $g_{l}=1, g_{s}=5.5856$ for protons and $g_{l}=0, g_{s}=-3.8263$ for neutrons. We are interested in the dipole operator $\left(\lambda=1, \mu^{\prime}=1\right)$. In cyclic coordinates it looks like

$$
\hat{O}_{11}=\mu_{N} \sqrt{\frac{3}{4 \pi}}\left[g_{s} \hat{S}_{1} / \hbar-g_{l} \sqrt{2} \sum_{\nu, \sigma} C_{1 \nu, 1 \sigma}^{11} r_{\nu} \nabla_{\sigma}\right]
$$

Its Wigner transformation is

$$
\left(O_{11}\right)_{W}=\mu_{N} \sqrt{\frac{3}{4 \pi}}\left[g_{s} \hat{S}_{1}-i \sqrt{2} g_{l} \sum_{\nu, \sigma} C_{1 \nu, 1 \sigma}^{11} r_{\nu} p_{\sigma}\right] / \hbar
$$


For the matrix element we have

$$
\begin{array}{r}
<\psi^{\prime}\left|\hat{O}_{11}\right| \psi^{\prime}>=\mu_{N} \sqrt{\frac{3}{4 \pi}}\left[g_{s} \hat{<} \psi^{\prime}\left|S_{1}\right| \psi^{\prime}>-i \sqrt{2} L_{11}^{\mathrm{p}+}\right] / \hbar \\
=\mu_{N} \sqrt{\frac{3}{2 \pi}}\left[-\frac{\hbar}{2}\left(g_{s}^{\mathrm{n}} F^{d \mathrm{n}}+g_{s}^{\mathrm{p}} F^{d \mathrm{p}}\right)-i L_{11}^{\mathrm{p}+}\right] / \hbar \\
=\mu_{N} \sqrt{\frac{3}{8 \pi}}\left[-\frac{1}{2}\left[\left(g_{s}^{\mathrm{n}}+g_{s}^{\mathrm{p}}\right) F^{d}+\left(g_{s}^{\mathrm{n}}-g_{s}^{\mathrm{p}}\right) \bar{F}^{d}\right]-\frac{i}{\hbar}\left(\mathcal{L}_{11}^{+}-\overline{\mathcal{L}}_{11}^{+}\right)\right] \\
=\mu_{N} \sqrt{\frac{3}{8 \pi}}\left[\frac{1}{2}\left(g_{s}^{\mathrm{p}}-g_{s}^{\mathrm{n}}\right) \bar{F}^{d}+\frac{i}{\hbar} \overline{\mathcal{L}}_{11}^{+}+\frac{i}{\hbar}\left[g_{s}^{\mathrm{n}}+g_{s}^{\mathrm{p}}-1\right] \mathcal{L}_{11}^{+}\right] .
\end{array}
$$

Deriving (39) we have used equilibrium relations (30) and $2 i L_{11}^{+}=-\hbar F^{d}$, which follows from the angular momentum conservation.

Due to the external field one of the dynamical equations of (32) becomes inhomogeneous:

$$
\dot{\overline{\mathcal{R}}}_{21}^{+}-\frac{2}{m} \overline{\mathcal{L}}_{21}^{+}+i \hbar \frac{\eta}{2}\left[\overline{\mathcal{R}}_{21}^{-}+2 \overline{\mathcal{R}}_{22}^{u}+2 \sqrt{\frac{3}{2}} \overline{\mathcal{R}}_{20}^{d}\right]=i \frac{3 \mu_{N}}{4 \hbar \sqrt{\pi}} R_{20}(e q) \exp ^{i \Omega t} .
$$

Solving the inhomogeneous set of equations (32) in the isovector and isoscalar cases one can find the required values of $\overline{\mathcal{L}}_{11}^{+}, \mathcal{L}_{11}^{+}$and $\bar{F}^{d}$ and calculate $\mathrm{B}(\mathrm{M} 1)$ factors for all (isovector and isoscalar) excitations.

To calculate the electric transition probability, it is necessary to excite the system with the external field operator

$$
\hat{O}_{2 \mu^{\prime}}=e r^{2} Y_{2 \mu^{\prime}}=\beta\{r \otimes r\}_{2 \mu^{\prime}},
$$

where $\beta=e \sqrt{\frac{15}{8 \pi}}$. Its Wigner transform is identical to $(41):\left(O_{2 \mu^{\prime}}\right)_{W}=\beta\{r \otimes r\}_{2 \mu^{\prime}}$. We consider the case $\mu^{\prime}=1$. The matrix element is given by

$$
<\psi^{\prime}\left|\hat{O}_{21}\right| \psi^{\prime}>=\beta R_{21}^{\mathrm{p}+}=\frac{1}{2} \beta\left(R_{21}^{+}-\bar{R}_{21}^{+}\right)=\frac{1}{2} \beta\left(\mathcal{R}_{21}^{+}-\overline{\mathcal{R}}_{21}^{+}\right) .
$$

The external field yields two inhomogeneous equations of (32):

$$
\begin{aligned}
\dot{\overline{\mathcal{L}}}_{21}^{+}- & \frac{1}{m} \overline{\mathcal{P}}_{21}^{+}+\left[m \omega^{2}-\frac{2}{\sqrt{3}} \alpha \chi_{0} R_{00}^{+}(e q)+\frac{1}{\sqrt{6}}(1+\alpha) \chi_{0} R_{20}^{+}(e q)\right] \overline{\mathcal{R}}_{21}^{+} \\
& +i \hbar \frac{\eta}{2}\left[\overline{\mathcal{L}}_{21}^{-}+2 \overline{\mathcal{L}}_{22}^{u}+2 \sqrt{\frac{3}{2}} \overline{\mathcal{L}}_{20}^{d}\right]=\frac{\beta}{\sqrt{3}}\left(\frac{\sqrt{2}}{4} R_{20}(e q)-R_{00}(e q)\right) \exp ^{i \Omega t}, \\
\dot{\mathcal{L}}_{11}^{+}+ & \sqrt{\frac{3}{2}}(1-\alpha) \chi_{0} R_{20}(e q) \overline{\mathcal{R}}_{21}^{+}+i \hbar \frac{\eta}{2}\left[\overline{\mathcal{L}}_{11}^{-}+\sqrt{2} \overline{\mathcal{L}}_{10}^{d}\right]=-\frac{\beta \sqrt{6}}{4} R_{20}(e q) \exp ^{i \Omega t} .
\end{aligned}
$$


Solving the inhomogeneous set of equations (32) in the isovector and isoscalar cases one can find the required values of $\overline{\mathcal{R}}_{21}^{+}$and $\mathcal{R}_{21}^{+}$and calculate $\mathrm{B}(\mathrm{E} 2)$ factors for all (isovector and isoscalar) excitations.

\subsection{Isovector excitations}

The energies and excitation probabilities obtained by the solution of the isovector set of equations (32) are given in Table 1. These results will be discussed by comparing them with some simplified cases, that will allows one to understand the physical nature of the considered excitations.

Tables 1, 2. Energies and excitation probabilities of the isovector system, calculated for ${ }^{164} \mathrm{Er}$ with two values of the spin orbit interaction constant $\eta$. The quantum numbers (including indices $+,-, u, d)$ of variables responsible for the generation of the present level are shown in a first column of Table 2 .

Table 1. $\eta=0.361 \mathrm{MeV}$

\begin{tabular}{c|c|c}
\hline$E_{\mathrm{iv}}, \mathrm{MeV}$ & $B(M 1), \mu_{N}^{2}$ & $B(E 2), B_{W}$ \\
\hline \hline 1.61 & 3.54 & 0.12 \\
2.18 & 5.33 & 1.02 \\
12.80 & 0.01 & 0.04 \\
14.50 & 0.01 & 0.03 \\
16.18 & 0.02 & 0.18 \\
16.20 & 0 & 0 \\
20.59 & 2.78 & 35.45 \\
$0.26 \mathrm{i}$ & $-5.43 \mathrm{i}$ & $0.0 \mathrm{i}$ \\
\hline
\end{tabular}

Table 2. $\eta=0$

\begin{tabular}{c||c|c|c}
\hline$(\lambda, \mu)^{s}$ & $E_{\mathrm{iv}}, \mathrm{MeV}$ & $B(M 1), \mu_{N}^{2}$ & $B(E 2), B_{W}$ \\
\hline \hline$(1,1)^{-}$ & 1.70 & 0 & 0 \\
$(1,1)^{+}$ & 2.07 & 9.03 & 1.17 \\
$(0,0)^{d}$ & 12.81 & 0 & 0 \\
$(2,1)^{-}$ & 14.50 & 0 & 0 \\
$(2,2)^{u}$ & 16.20 & 0 & 0 \\
$(2,0)^{d}$ & 16.20 & 0 & 0 \\
$(2,1)^{+}$ & 20.55 & 2.81 & 35.70 \\
$(1,0)^{d}$ & 0 & - & - \\
\hline
\end{tabular}

\subsubsection{The limit $\eta=0$}

In the case of vanishing spin orbit potential, $\eta=0$, the set of equations (32) splits into four independent subsets.

The first one is

$$
\dot{\overline{\mathcal{L}}}_{21}^{+}=\frac{1}{m} \overline{\mathcal{P}}_{21}^{+}-\left[m \omega^{2}-\frac{2}{\sqrt{3}} \alpha \chi_{0} R_{00}(e q)+\frac{1}{\sqrt{6}}(1+\alpha) \chi_{0} R_{20}(e q)\right] \overline{\mathcal{R}}_{21}^{+},
$$




$$
\begin{aligned}
\dot{\overline{\mathcal{L}}}_{11}^{+} & =-\sqrt{\frac{3}{2}}(1-\alpha) \chi_{0} R_{20}(e q) \overline{\mathcal{R}}_{21}^{+}, \\
\dot{\mathcal{\mathcal { R }}}_{21}^{+} & =\frac{2}{m} \overline{\mathcal{L}}_{21}^{+}, \\
\dot{\overline{\mathcal{P}}}_{21}^{+} & =-\left[2 m \omega^{2}+\sqrt{\frac{2}{3}} \chi_{0} R_{20}(e q)\right] \overline{\mathcal{L}}_{21}^{+}+\sqrt{6} \chi_{0} R_{20}(e q) \overline{\mathcal{L}}_{11}^{+} .
\end{aligned}
$$

This set of equations for spin-scalar variables coincides with the set of equations (29) of [16] and describes the joint dynamics of the "standard" nuclear scissors mode and the $\mu=1$ branch of IVGQR. Remembering that $\chi_{0}=6 \kappa_{0}, R_{2 \mu}=Q_{2 \mu} / \sqrt{6}, Q_{20}=Q_{00} \frac{4}{3} \delta$ and taking the self consistent value $\kappa_{0}=-\frac{m \bar{\omega}^{2}}{4 Q_{00}}[17]$ we find the energies of these modes

$$
\Omega_{ \pm}^{2}=\bar{\omega}^{2}(2-\alpha)(1+\delta / 3) \pm \sqrt{\bar{\omega}^{4}(2-\alpha)^{2}(1+\delta / 3)^{2}-4 \bar{\omega}^{4}(1-\alpha) \delta^{2}} .
$$

Usually [16] we choose $\alpha=-2$. Then

$$
\Omega_{ \pm}^{2}=4 \bar{\omega}^{2}\left(1+\frac{\delta}{3} \pm \sqrt{\left(1+\frac{\delta}{3}\right)^{2}-\frac{3}{4} \delta^{2}}\right) .
$$

The second subset reads

$$
\begin{aligned}
& \dot{\overline{\mathcal{L}}}_{21}^{-}=\frac{1}{m} \overline{\mathcal{P}}_{21}^{-}-\left[m \omega^{2}+\frac{1}{\sqrt{6}} \chi_{0} R_{20}(e q)\right] \overline{\mathcal{R}}_{21}^{-}, \\
& \dot{\overline{\mathcal{L}}}_{11}^{-}=-\sqrt{\frac{3}{2}} \chi_{0} R_{20}(e q) \overline{\mathcal{R}}_{21}^{-}, \\
& \dot{\overline{\mathcal{R}}}_{21}^{-}=\frac{2}{m} \overline{\mathcal{L}}_{21}^{-} \text {, } \\
& \dot{\overline{\mathcal{P}}}_{21}^{-}=-\left[2 m \omega^{2}+\sqrt{\frac{2}{3}} \chi_{0} R_{20}(e q)\right] \overline{\mathcal{L}}_{21}^{-}+\sqrt{6} \chi_{0} R_{20}(e q) \overline{\mathcal{L}}_{11}^{-} .
\end{aligned}
$$

In the absence of spin dependent forces one could naively expect that this set of equations should be identical with (44). However we observe an essential difference between the two, the difference being determined by the variation of the mean field (the variable $Z_{21}^{+}$). This fact can easily be understood by comparing the first and second equations of (27). The terms responsible for the contribution of the mean field are $\left\{Z_{2}^{+} \otimes R_{2}^{+}\right\}_{\lambda \mu}$ and $\left\{Z_{2}^{+} \otimes R_{2}^{-}\right\}_{\lambda \mu}$. Their variations are $\left\{\delta Z_{2}^{+} \otimes R_{2}^{+}(e q)+Z_{2}^{+}(e q) \otimes \delta R_{2}^{+}\right\}_{\lambda \mu}$ and $\left\{\delta Z_{2}^{+} \otimes R_{2}^{-}(e q)+Z_{2}^{+}(e q) \otimes \delta R_{2}^{-}\right\}_{\lambda \mu}$ respectively. We assumed that $R_{2 \mu}^{-}(e q)=0$, so the contribution of the mean field variation $\delta Z_{2 \mu}^{+}$in the second equation disappears and we arrive to the announced difference. In other words, when the 
particles with different directions of spins move in phase, the mean field variations generated by their motion add and, when they move out of phase, the respective mean field variations annihilate one another.

The set of equations for spin-vector variables (47) has the following eigenfrequencies:

$$
\Omega_{ \pm}^{2}=2 \bar{\omega}^{2}\left(1+\frac{\delta}{3} \pm \sqrt{\left(1+\frac{\delta}{3}\right)^{2}-\delta^{2}}\right)
$$

The numerical estimates for ${ }^{164} E r$ are shown in Table 2. The low lying level has $E_{11}^{-}(l o w)=$ $\hbar \Omega_{-}=1.70 \mathrm{MeV}$ and the high lying level has $E_{21}^{-}($high $)=\hbar \Omega_{+}=14.50 \mathrm{MeV}$. So, in addition to the well known ("standard") scissors mode with energy $E_{11}^{+}=2.07 \mathrm{MeV}$, describing the relative rotational vibrations of all (spin up + spin down) neutrons with respect of all protons, we get one more scissors mode, which is generated by the new type of the nuclear collective motion the relative rotational vibrations of spin up nucleons with respect of spin down nucleons. The inclusion of the spin orbital interaction only slightly shifts these levels apart (compare Tables 1 and 2$): 1.70 \rightarrow 1.61$ and $2.07 \rightarrow 2.18$. However, the main role of the spin orbital interaction consists in the excitation of the new scissors mode. This mode is not excited directly by the electromagnetic field (see formula (40)). It can be excited only indirectly, via the coupling with the "standard" scissors mode by means of the spin orbital interaction.

The new scissors mode is generated by the variable $\overline{\mathcal{L}}_{11}^{-}$responsible for the relative rotational motion. It is easy to see by removing this variable from the set of equations (47). In this case its characteristic equation gives only one level with energy $E=2 \hbar \bar{\omega} \sqrt{1+\delta / 3}$, which practically coincides with $E_{21}^{-}($high) and lies in between the isoscalar and isovector giant quadrupole resonances. From the physical interpretation of variables $\overline{\mathcal{R}}_{21}^{-}, \overline{\mathcal{P}}_{21}^{-}, \overline{\mathcal{L}}_{21}^{-}$, responsible for this mode, it follows that it describes rather complicate motion: the proton system oscillates out of phase with the neutron system, whereas inside of each system spin up nucleons oscillate out of phase with spin down nucleons. In the absence of the proper residual interaction it has the standard shell model value of the energy $E=2 \hbar \omega(\delta)$. So, the new scissors mode is accompanied by the high lying excitation, which can be called the spin-vector giant quadrupole resonance. As one can see, the situation is quite similar to that of the "standard" scissors mode which exists only together [16] with isovector giant quadrupole resonance. 
Let us point to the interesting feature of the new scissors mode. As one knows, the variable $\overline{\mathcal{L}}_{11}^{-}$is spin-vector (see the text after eq. (25)), i.e. it describes the relative rotational oscillations of spin up nucleons with respect of spin down nucleons (spin scissors). This means that we have proton spin scissors and neutron spin scissors. At the same time $\overline{\mathcal{L}}_{11}^{-}$is the isovector variable, i.e. it describes the relative rotational oscillations of protons with respect of neutrons. So, the resulting motion described by the variable $\overline{\mathcal{L}}_{11}^{-}$looks rather complex - proton spin scissors vibrate in a rotational way (like scissors) with respect of neutron spin scissors, i.e. we have the scissors made of two other scissors!

It is necessary also to stress the important role of the Fermi surface deformation (variable $\left.\overline{\mathcal{P}}_{21}^{-}\right)$. Here the situation is exactly the same as in the case of the "standard" scissors mode. If one removes the variable $\overline{\mathcal{P}}_{21}^{-}$from the set of equations (47), one gets a zero value for $E_{11}^{-}($low $)$ and a considerably changed expression for the high lying mode $-E_{21}^{-}(h i g h)=\hbar \bar{\omega} \sqrt{2(1+\delta / 3)}$.

\section{The third subset reads}

$$
\begin{aligned}
& \dot{\overline{\mathcal{L}}}_{22}^{u}=\frac{1}{m} \overline{\mathcal{P}}_{22}^{u}-\left[m \omega^{2}-\sqrt{\frac{2}{3}} \chi_{0} R_{20}(e q)\right] \overline{\mathcal{R}}_{22}^{u}, \\
& \dot{\overline{\mathcal{R}}}_{22}^{u}=\frac{2}{m} \overline{\mathcal{L}}_{22}^{u}, \\
& \dot{\overline{\mathcal{P}}}_{22}^{u}=-\left[2 m \omega^{2}-2 \sqrt{\frac{2}{3}} \chi_{0} R_{20}(e q)\right] \overline{\mathcal{L}}_{22}^{u} .
\end{aligned}
$$

Its solution gives $E_{22}=2 \hbar \bar{\omega} \sqrt{\left(1+\frac{4}{3} \delta\right)}$.

\section{The fourth subset reads}

$$
\begin{aligned}
& \dot{\overline{\mathcal{L}}}_{20}^{d}=\frac{1}{m} \overline{\mathcal{P}}_{20}^{d}-\left[m \omega^{2}+\sqrt{\frac{2}{3}} \chi_{0} R_{20}(e q)\right] \overline{\mathcal{R}}_{20}^{d}+\frac{2}{\sqrt{3}} \chi_{0} R_{20}(e q) \overline{\mathcal{R}}_{00}^{d}, \\
& \dot{\overline{\mathcal{R}}}_{20}^{d}=\frac{2}{m} \overline{\mathcal{L}}_{20}^{d}, \\
& \dot{\overline{\mathcal{P}}}_{20}^{d}=-\left[2 m \omega^{2}+2 \sqrt{\frac{2}{3}} \chi_{0} R_{20}(e q)\right] \overline{\mathcal{L}}_{20}^{d}+\frac{4}{\sqrt{3}} \chi_{0} R_{20}(e q) \overline{\mathcal{L}}_{00}^{d}, \\
& \dot{\overline{\mathcal{L}}}_{00}^{d}=\frac{1}{m} \overline{\mathcal{P}}_{00}^{d}-m \omega^{2} \overline{\mathcal{R}}_{00}^{d}+\frac{2}{\sqrt{3}} \chi_{0} R_{20}(e q) \overline{\mathcal{R}}_{20}^{d}, \\
& \dot{\overline{\mathcal{R}}}_{00}^{d}=\frac{2}{m} \overline{\mathcal{L}}_{00}^{d}, \\
& \dot{\overline{\mathcal{P}}}_{00}^{d}=-2 m \omega^{2} \overline{\mathcal{L}}_{00}^{d}+\frac{4}{\sqrt{3}} \chi_{0} R_{20}(e q) \overline{\mathcal{L}}_{20}^{d} .
\end{aligned}
$$

Its solution gives $E_{20}=2 \hbar \bar{\omega} \sqrt{\left(1+\frac{4}{3} \delta\right)}$ and $E_{00}=2 \hbar \bar{\omega} \sqrt{\left(1-\frac{2}{3} \delta\right)}$. 
According to the physical interpretation of variables of the third and fourth subsets they describe the spin-flip modes: giant quadrupole and monopole spin-flip resonances with energies $E_{20}=E_{22}=16.20 \mathrm{MeV}$ and $E_{00}=12.81 \mathrm{MeV}$ respectively (Table 2). Our Hamiltonian does not contain any forces, which can change the direction of the spin, so these modes have zero values of excitation probabilities and the standard shell model values of energies $E=2 \hbar \omega(\delta)$. The spin orbital interaction changes this result only negligibly.

And finally, a few words about the solution with the imaginary eigenfrequency (last line of Table 1). From where does it appear? We carefully checked that it is not a mistake of calculation or computation, because the energy weighted sum rule (see Appendix C) is fulfilled, the contribution of this unexpected mode being $\sim 1.5 \%$. What could be its physical interpretation? The time dependence of variables in this mode is $e^{-\Omega_{0} t}\left(\Omega_{0}=0.26 \mathrm{MeV}\right)$, i.e. this excitation is unstable, i.e. decaying. This may be connected with the neglect by higher order moments. One can hope also that the inclusion of the spin-spin or spin-multipole residual interaction will produce the necessary restoring force and stabilize the mode.

\subsubsection{The approximation $f^{\downarrow \uparrow}=f^{\uparrow \downarrow}=0$}

The set of equations for the variables $X^{ \pm}$is obtained from (32) by setting variables $X^{u}, X^{d}$ to zero. Its solution gives energies and excitation probabilities given in the Table 3 . The comparison with the exact solution shows that the approximation of two (spin up and spin down) "liquids" can be used with great care - it works very well to describe energies and $B(E 2)$ factors, but leads to erroneous results for $B(M 1)$ factors because the essential contribution into their values stems from the (now neglected) variable $\bar{F}^{d}$.

Table 3. Energies and excitation probabilities of the isovector system, calculated for ${ }^{164} \mathrm{Er}$ with $\eta=0.361 \mathrm{MeV}$ in the approximation $f^{\downarrow \uparrow}=f^{\uparrow \downarrow}=0$.

\begin{tabular}{c|c|c}
\hline$E_{\text {iv }}, \mathrm{MeV}$ & $B(M 1), \mu_{N}^{2}$ & $B(E 2), B_{W}$ \\
\hline \hline 1.62 & 1.30 & 0.16 \\
2.15 & 7.74 & 1.01 \\
14.50 & 0 & 0.03 \\
20.59 & 2.81 & 35.66 \\
\hline
\end{tabular}




\subsection{Isoscalar case}

The energies and excitation probabilities obtained by the solution of the isoscalar set of equations (32) are demonstrated in the Table 4. Analogously to the isovector case the results will be discussed in comparison with $\eta=0$ limit, what helps to understand their physical nature.

Tables 4, 5. Energies and excitation probabilities of the isoscalar system, calculated for ${ }^{164} \mathrm{Er}$ with two values of the spin orbital interaction constant $\eta$. The quantum numbers (including indices $+,-, u, d$ ) of variables responsible for the generation of the present level are shown in a first column of Table 5.

Table 4. $\eta=0.361 \mathrm{MeV}$

\begin{tabular}{c|c|c}
\hline$E_{\text {is }}, \mathrm{MeV}$ & $B(M 1), \mu_{N}^{2}$ & $B(E 2), B_{W}$ \\
\hline \hline 1.73 & -0.07 & 1.12 \\
0.39 & 0.24 & 117.19 \\
12.83 & 0 & 0.66 \\
14.51 & 0 & 0.12 \\
16.20 & 0 & 0 \\
16.22 & 0 & 0.20 \\
10.28 & 0 & 66.50 \\
$0.20 \mathrm{i}$ & $-0.12 \mathrm{i}$ & $30.30 \mathrm{i}$ \\
\hline
\end{tabular}

Table 5. $\eta=0$

\begin{tabular}{c||c|c|c}
\hline$(\lambda, \mu)^{s}$ & $E_{\mathrm{is}}, \mathrm{MeV}$ & $B(M 1), \mu_{N}^{2}$ & $B(E 2), B_{W}$ \\
\hline \hline$(1,1)^{-}$ & 1.70 & 0 & 0 \\
$(1,1)^{+}$ & 0 & - & - \\
$(0,0)^{d}$ & 12.81 & 0 & 0 \\
$(2,1)^{-}$ & 14.51 & 0 & 0 \\
$(2,2)^{u}$ & 16.20 & 0 & 0 \\
$(2,0)^{d}$ & 16.20 & 0 & 0 \\
$(2,1)^{+}$ & 10.33 & 0 & 67.47 \\
$(1,0)^{d}$ & 0 & - & - \\
\hline
\end{tabular}

\subsubsection{The limit of vanishing spin-orbit potential $(\eta=0)$}

In the isoscalar case the set of equations (44) is transformed into

$$
\begin{aligned}
\dot{\mathcal{L}}_{21}^{+} & =\frac{1}{m} \mathcal{P}_{21}^{+}-\left[m \omega^{2}-\frac{2}{\sqrt{3}} \chi_{0} R_{00}(e q)+\frac{2}{\sqrt{6}} \chi_{0} R_{20}(e q)\right] \mathcal{R}_{21}^{+}, \\
\dot{\mathcal{L}}_{11}^{+} & =0 \\
\dot{\mathcal{R}}_{21}^{+} & =\frac{2}{m} \mathcal{L}_{21}^{+}, \\
\dot{\mathcal{P}}_{21}^{+} & =-\left[2 m \omega^{2}+\sqrt{\frac{2}{3}} \chi_{0} R_{20}(e q)\right] \mathcal{L}_{21}^{+}+\sqrt{6} \chi_{0} R_{20}(e q) \mathcal{L}_{11}^{+} .
\end{aligned}
$$

These equations coincide with the set of equations (24) of [16]. They describe the joint dynamics

of the $\mu=1$ branch of ISGQR and the orbital angular momentum (its $l_{1}$ projection). In the 
absence of spin the orbital angular momentum is conserved $\left(\frac{d}{d t}<\hat{l}_{1}>=-i \sqrt{2} \dot{\mathcal{L}}_{11}^{+}=0\right)$ as it should be. However, in the case with spin the situation is changed radically. Now the orbital angular momentum and spin can vibrate out of phase keeping their sum (the total angular momentum) unchanged. This new circumstance reveals itself in the appearance of the new low lying mode which describes the relative motion of the orbital angular momentum and spin of the nucleus (see Table 4). According to our calculations this mode $(\mathrm{E}=0.39 \mathrm{MeV})$ has essentially electric character with $B(E 2)=117 \mathrm{~W} . u$. demonstrating also a small admixture of the magnetic properties with $B(M 1)=0.24 \mu_{N}^{2}$.

As it is seen, the set of equations (47) does not depend on $\alpha$, so it is not changed in the isoscalar case. Naturally it has just the same eigenvalues: $E($ low $)=1.70 \mathrm{MeV}, E($ high $)=14.50$ $\mathrm{MeV}$. The collective motion corresponding to these two modes is more simple, than in the isovector case. The variable $\mathcal{L}_{11}^{-}$, responsible for the low lying excitation, describes the real spin scissors: all spin up nucleons (protons together with neutrons) oscillate rotationally out of phase with all spin down nucleons. This mode has $\mathrm{B}(\mathrm{M} 1)=0$ because magnetic moments produced by two parts of spin scissors annihilate one another. The variables $\mathcal{R}_{21}^{-}, \mathcal{P}_{21}^{-}, \mathcal{L}_{21}^{-}$, responsible for the high lying excitation, describe out of phase oscillations of all spin up nucleons (protons together with neutrons) with respect of all spin down nucleons. The inclusion of the spin orbital interaction influences practically only on the low-lying mode - its energy becomes a little bit higher $(1.70 \mathrm{MeV} \rightarrow 1.73 \mathrm{MeV})$ and it acquires the nonzero $\mathrm{B}(\mathrm{E} 2)$ value $(\mathrm{B}(\mathrm{E} 2)=1.12$ W.u.) demonstrating its electric character. The negligibly small negative B(M1) value and the imaginary solution (last line of the Table 4) appear, probably, due to the same reasons, as was already discussed at the end of section 5.1.1.

\subsubsection{The approximation $f^{\downarrow \uparrow}=f^{\uparrow \downarrow}=0$}

Removing the variables $X^{u}, X^{d}$ from (32) with $\alpha=1$ we obtain the isoscalar set of equations for $X^{ \pm}$variables. The results of calculations are shown in the Table 6 . It is readily seen, that approximate results reproduce rather well the exact ones (Table 4) except for one case: instead of the excitation with the energy $\mathrm{E}=0.39 \mathrm{MeV}$, which is generated by the relative motion of the orbital angular momentum and the spin, we obtain an imaginary solution. This is not 
surprising, since after removing the variable $F^{d}$, one can not ensure the conservation of the total angular momentum (see section 4.3).

Table 6. Energies and excitation probabilities of the isoscalar system, calculated for ${ }^{164} \mathrm{Er}$ with $\eta=0.361 \mathrm{MeV}$ in the approximation $f^{\downarrow \uparrow}=f^{\uparrow \downarrow}=0$.

\begin{tabular}{c|c|c}
\hline$E_{\mathrm{iv}}, \mathrm{MeV}$ & $B(M 1), \mu_{N}^{2}$ & $B(E 2), B_{W}$ \\
\hline \hline $0.18 \mathrm{i}$ & $0.79 \mathrm{i}$ & $-212.4 \mathrm{i}$ \\
1.72 & 0.08 & 0.99 \\
10.32 & 0 & 67.34 \\
14.51 & 0 & 0.12 \\
\hline
\end{tabular}

\section{Conclusion}

In this work, the WFM method is applied for a first time to solve the TDHF equation including spin dynamics. The model Hamiltonian consists of a harmonic oscillator with spin orbit mean field potential plus quadrupole-quadrupole residual interaction. Spin dependent collective variables are defined and a corresponding closed set of nonlinear dynamical equations is set up. The equations are solved in small amplitude approximation. Two isovector and two isoscalar low lying eigenfrequencies and five isovector and five isoscalar high lying eigenfrequencies have been found. Three low lying levels correspond to the excitation of a new kind of mode, unknown earlier. For example the isovector level with energy $\mathrm{E}=1.61 \mathrm{MeV}$ describes the rotational oscillations of nucleons with the spin projection "up" with respect of nucleons with the spin projection "down", i.e. one can talk of a nuclear spin scissors mode. Thus the experimentally observed group of $1^{+}$peaks in the interval 2-4 MeV, associated usually with the nuclear scissors mode, in reality consists of the excitations of the "spin" scissors mode together with the "standard" scissors mode. It is necessary to point out an interesting feature of this new mode. Being of isovector nature, it describes also the motion of protons with respect to neutrons and, therefore, the resulting motion can be characterized as the rotational oscillations of proton spin scissors with respect of neutron spin scissors. In the analogous isoscalar mode with the energy $\mathrm{E}=1.73 \mathrm{MeV}$ the proton and neutron spin scissors move in phase. This mode has $\mathrm{B}(\mathrm{M} 1)=0$ because magnetic moments produced by two different parts of proton spin scissors annihilate 
each other. A small B(E2) factor is explained by the coupling with the ISGQR.

One more new low lying mode with energy $\mathrm{E}=0.39 \mathrm{MeV}$ is generated by the out of phase oscillations of the orbital angular momentum and spin of the nucleus. The rather big B(E2) factor of this mode still waits for its explanation.

There are ten high lying excitations, the two of them being really new: isovector and isoscalar "spin-vector" resonances with energies E=14.5 MeV. Further six high lying modes can be interpreted as spin-flip ones. In the absence of residual interactions all these eight modes have very small excitation probabilities and standard shell model energies $\mathrm{E}=2 \hbar \omega(\delta)$.

In the light of the above results, the study of all discussed (low and high lying) excitations with proper residual interactions included will be the natural continuation of this work. It is known very well that pairing is very important for the correct description of the "standard" scissors. Therefore one has to take into account pair correlations too, i.e. to move from the TDHF equations to TDHFB equations. This also will be a task for the future.

\section{Acknowledgments}

The useful discussions with M. Urban are gratefully acknowledged.

\section{Appendix A}

According to the definition [13] of Wigner transformation one finds

$$
\begin{array}{r}
h_{l_{0}}(\mathbf{r}, \mathbf{p})=\int d^{3} s e^{-i \mathbf{p s} / \hbar} h_{l_{0}}\left(\mathbf{r}+\frac{\mathbf{s}}{2}, \mathbf{r}-\frac{\mathbf{s}}{2}\right) \\
=-\frac{\hbar}{2} \eta(\mathbf{r})\left(x p_{y}-y p_{x}\right)-\frac{i \hbar}{2}\left(x \frac{\partial}{\partial y}-y \frac{\partial}{\partial x}\right) \frac{\hbar}{2} \eta(\mathbf{r}) .
\end{array}
$$

Usually we will take $\eta(\mathbf{r})=\eta(r)$. Then $\frac{\partial}{\partial x_{i}} \eta(\mathbf{r})=\frac{x_{i}}{r} \frac{\partial \eta}{\partial r}$. As a result $\left(x \frac{\partial}{\partial y}-y \frac{\partial}{\partial x}\right) \eta(\mathbf{r})=0$ and

$$
h_{l_{0}}(\mathbf{r}, \mathbf{p})=-\frac{\hbar}{2} \eta(r)\left(x p_{y}-y p_{x}\right)
$$

In a similar way one finds

$$
\begin{aligned}
h_{l_{1}}(\mathbf{r}, \mathbf{p}) & =\frac{\hbar}{2} \eta(r)\left[i\left(x p_{z}-z p_{x}\right)-\left(y p_{z}-z p_{y}\right)\right], \\
h_{l_{-1}}(\mathbf{r}, \mathbf{p}) & =-\frac{\hbar}{2} \eta(r)\left[i\left(x p_{z}-z p_{x}\right)+\left(y p_{z}-z p_{y}\right)\right] .
\end{aligned}
$$


The Wigner transformation of a product of two operators is given [13] by the following formula

$$
\begin{aligned}
(\hat{h} \hat{\rho})_{W} & =h(\mathbf{r}, \mathbf{p}) \exp \left(\frac{i \hbar}{2} \stackrel{\leftrightarrow}{\Lambda}\right) f(\mathbf{r}, \mathbf{p}) \\
& =h(\mathbf{r}, \mathbf{p}) f(\mathbf{r}, \mathbf{p})+\frac{i \hbar}{2}\{h, f\}-\frac{\hbar^{2}}{8}\{\{h, f\}\}+o\left(\hbar^{3}\right)
\end{aligned}
$$

where $\overleftrightarrow{\Lambda}=\overleftarrow{\nabla}_{r} \vec{\nabla}_{p}-\overleftarrow{\nabla}_{p} \vec{\nabla}_{r}, \quad\{h, f\}=h \overleftrightarrow{\Lambda} f$ is the Poisson bracket of functions $h(\mathbf{r}, \mathbf{p})$ and $f(\mathbf{r}, \mathbf{p}), \quad\{\{h, f\}\}=h(\mathbf{r}, \mathbf{p})(\overleftrightarrow{\Lambda})^{2} f(\mathbf{r}, \mathbf{p})$ is their double Poisson bracket.

\section{Appendix B}

\section{Integrals of motion.}

$$
\begin{aligned}
\overline{\mathcal{L}}_{11}^{+} & =-i \frac{2(1-\alpha) \chi_{0}}{\hbar \eta R_{20}^{+}(e q)} \sqrt{\frac{3}{2}} C^{d}+i \frac{\hbar}{2} \bar{F}^{d}, \\
\overline{\mathcal{P}}_{20}^{d} & =m\left(m \omega^{2}-\sqrt{\frac{2}{3}} \chi_{0} R_{20}^{+}(e q)\right)\left(\sqrt{\frac{3}{2}} \bar{R}_{22}^{u}-\sqrt{2} \bar{R}_{00}^{d}-\bar{R}_{20}^{d}\right)+\sqrt{\frac{3}{2}} \bar{P}_{22}^{u}-\sqrt{2} \bar{P}_{00}^{d}, \\
\overline{\mathcal{L}}_{21}^{+} & =i \frac{2}{\hbar \eta}\left[\frac{1}{m} \bar{P}_{22}^{u}+\left(m \omega^{2}-\sqrt{\frac{2}{3}} \chi_{0} R_{20}^{+}(e q)\right)\left(\bar{R}_{22}^{u}-C^{d}\right)\right] \\
& -i \frac{2}{\hbar \eta}\left(m \omega^{2}-\frac{2 \alpha \chi_{0}}{\sqrt{3}} R_{00}^{+}(e q)+\frac{(1+\alpha) \chi_{0}}{\sqrt{6}} R_{20}^{+}(e q)\right) C^{d} \\
& \left.-i \frac{\hbar \eta m}{4}\left[\left(\bar{R}_{21}^{-}+2 \bar{R}_{22}^{u}-3 C^{d}\right)\right)+\frac{3 \sqrt{2}}{m \chi_{0} R_{20}^{+}(e q)}\left(\bar{P}_{00}^{d}+m \omega^{2} \bar{R}_{00}^{d}\right)\right], \text { where } \\
C^{d} & \equiv \sqrt{\frac{2}{3}} \bar{R}_{20}^{d}-\frac{\left(\bar{P}_{00}^{d}+m \omega^{2} \bar{R}_{00}^{d}\right)}{\sqrt{2} m \chi_{0} R_{20}^{+}(e q)} .
\end{aligned}
$$

\section{Appendix $\mathrm{C}$}

\section{The sum rule for magnetic transitions.}

Magnetic operator

$$
\hat{O}_{1 \phi}=\sqrt{\frac{3}{4 \pi}}\left(g_{l} \hat{l}_{\phi}+g_{s} \hat{S}_{\phi}\right) \mu_{N} / \hbar
$$

obeys the following sum rule

$$
\sum_{\nu}\left(E_{\nu}-E_{0}\right)\left(|<\nu| \hat{O}_{11}|0>|^{2}+|<\nu| \hat{O}_{1-1}|0>|^{2}\right)=-<0\left|\left[\hat{O}_{11},\left[H, \hat{O}_{1-1}\right]\right]\right| 0>.
$$


The oscillator part $h_{o s c}$ of the Hamiltonian (6) commutes with $\hat{S}_{\phi}$, so we can use for the double commutator of $\hat{O}_{1 \phi}$ with $h_{\text {osc }}$ the result found in [16]:

$$
\left[\hat{O}_{1 \phi},\left[h_{o s c}, \hat{O}_{1 \phi^{\prime}}\right]\right]=\frac{15}{2 \pi} \bar{\chi} \sum_{i}^{Z} \sum_{j}^{N} \sum_{\nu, \sigma, \epsilon}(-1)^{\nu} C_{2 \nu, 2 \sigma}^{1 \phi} C_{2-\nu, 2 \epsilon}^{1 \phi^{\prime}}\left\{r_{i} \otimes r_{i}\right\}_{2 \epsilon}\left\{r_{j} \otimes r_{j}\right\}_{2 \sigma} \mu_{N}^{2} .
$$

Its ground state matrix element is [16]

$$
<0\left|\left[\hat{O}_{11},\left[h_{o s c}, \hat{O}_{1-1}\right]\right]\right| 0>=\frac{9}{16 \pi} \bar{\chi}\left(R_{20}^{e q}\right)^{2} \mu_{N}^{2}=-\frac{1-\alpha}{4 \pi} Q_{00} m \bar{\omega}^{2} \delta^{2} \mu_{N}^{2}
$$

Now we have to calculate the double commutator of $\hat{O}_{1 \phi}$ with the spin orbital part $h_{l s}$ of the Hamiltonian (6). One has

$$
\begin{aligned}
& \hat{h}_{l s}=-\eta(\mathbf{r}) \sum_{\mu=-1}^{1}(-1)^{\mu} \hat{l}_{\mu} \hat{S}_{-\mu}, \quad\left[\hat{l}_{\mu}, \hat{l}_{\phi}\right]=-\sqrt{2} C_{1 \mu, 1 \phi}^{1 \nu} \hat{l}_{\nu}, \quad\left[\hat{S}_{\mu}, \hat{S}_{\phi}\right]=-\sqrt{2} C_{1 \mu, 1 \phi}^{1 \nu} \hat{S}_{\nu} \\
& {\left[h_{l s}, \hat{l}_{\phi}\right]=-\eta(\mathbf{r}) \sum_{\mu=-1}^{1}(-1)^{\mu}\left[\hat{l}_{\mu}, \hat{l}_{\phi}\right] \hat{S}_{-\mu}=\sqrt{2} \eta(\mathbf{r}) \sum_{\mu=-1}^{1}(-)^{\mu} C_{1 \mu, 1 \phi}^{1 \nu} \hat{l}_{\nu} \hat{S}_{-\mu}} \\
& {\left[h_{l s}, \hat{S}_{\phi}\right]=-\eta(\mathbf{r}) \sum_{\mu=-1}^{1}(-1)^{\mu} \hat{l}_{\mu}\left[\hat{S}_{-\mu}, \hat{S}_{\phi}\right]=\sqrt{2} \eta(\mathbf{r}) \sum_{\mu=-1}^{1}(-)^{\mu} C_{1-\mu, 1 \phi}^{1 \nu} \hat{l}_{\mu} \hat{S}_{\nu}} \\
& {\left[h_{l s}, \hat{O}_{1 \phi}\right]=\frac{\mu_{N}}{\hbar} \sqrt{\frac{3}{2 \pi}} \eta(\mathbf{r}) \sum_{\mu=-1}^{1}(-1)^{\mu} C_{1 \mu, 1 \phi}^{1 \nu}\left(g_{l} \hat{l}_{\nu} \hat{S}_{-\mu}+g_{s} \hat{l}_{-\mu} \hat{S}_{\nu}\right)} \\
& {\left[\hat{l}_{\phi},\left[h_{l s}, \hat{O}_{1 \phi^{\prime}}\right]\right]=-\frac{\mu_{N}}{\hbar} \sqrt{\frac{3}{\pi}} \eta(\mathbf{r}) \sum_{\mu=-1}^{1}(-1)^{\mu} C_{1 \mu, 1 \phi^{\prime}}^{1 \nu^{\prime}}\left(g_{l} C_{1 \phi, 1 \nu^{\prime}}^{1 \nu} \hat{l}_{\nu} \hat{S}_{-\mu}+g_{s} C_{1 \phi, 1-\mu}^{1 \nu} \hat{l}_{\nu} \hat{S}_{\nu^{\prime}}\right)} \\
& =-3 \frac{\mu_{N}}{\hbar} \sqrt{\frac{3}{\pi}} \eta(\mathbf{r})\left(g_{l}-g_{s}\right) \sum_{j, m}\left\{\begin{array}{l}
111 \\
j 11
\end{array}\right\} C_{1 \phi^{\prime}, 1 \phi}^{j m}\{\hat{l} \otimes \hat{S}\}_{j m} \\
& {\left[\hat{S}_{\phi},\left[h_{l s}, \hat{O}_{1 \phi^{\prime}}\right]\right]=-\frac{\mu_{N}}{\hbar} \sqrt{\frac{3}{\pi}} \eta(\mathbf{r}) \sum_{\mu=-1}^{1}(-1)^{\mu} C_{1 \mu, 1 \phi^{\prime}}^{1 \nu^{\prime}}\left(g_{l} C_{1 \phi, 1-\mu}^{1 \nu} \hat{l}_{\nu^{\prime}} \hat{S}_{\nu}+g_{s} C_{1 \phi, 1 \nu^{\prime}}^{1 \nu} \hat{l}_{-\mu} \hat{S}_{\nu}\right)} \\
& =3 \frac{\mu_{N}}{\hbar} \sqrt{\frac{3}{\pi}} \eta(\mathbf{r})\left(g_{l}-g_{s}\right) \sum_{j, m}\left\{\begin{array}{lll}
1 & 1 & 1 \\
j & 1 & 1
\end{array}\right\}(-1)^{j} C_{1 \phi^{\prime}, 1 \phi}^{j m}\{\hat{l} \otimes \hat{S}\}_{j m}, \\
& {\left[\hat{O}_{\phi},\left[h_{l s}, \hat{O}_{1 \phi^{\prime}}\right]\right]=-\frac{\mu_{N}^{2}}{\hbar^{2}} \frac{9}{2 \pi} \eta(\mathbf{r}) \sum_{i=1}^{A}\left(g_{l}^{i}-g_{s}^{i}\right) \sum_{j, m}\left[g_{l}^{i}-g_{s}^{i}(-1)^{j}\right]\left\{\begin{array}{l}
111 \\
j 11
\end{array}\right\} C_{1 \phi^{\prime}, 1 \phi}^{j m}\left\{\hat{l}^{i} \otimes \hat{S}^{i}\right\}_{j m} .}
\end{aligned}
$$


We need the ground state matrix element of this double commutator for $\phi=1, \phi^{\prime}=-1$ :

$$
\begin{aligned}
<0\left|\left[\hat{O}_{11},\left[h_{l s}, \hat{O}_{1-1}\right]\right]\right| 0>= & -\frac{\mu_{N}^{2}}{\hbar^{2}} \frac{9}{2 \pi} \sum_{i=1}^{A}\left(g_{l}^{i}-g_{s}^{i}\right) \sum_{j}\left[g_{l}^{i}-g_{s}^{i}(-1)^{j}\right]\left\{\begin{array}{lll}
1 & 1 & 1 \\
j & 1 & 1
\end{array}\right\} \\
& C_{1-1,11}^{j 0}<0\left|\eta(\mathbf{r})\left\{\hat{l}^{i} \otimes \hat{S}^{i}\right\}_{j 0}\right| 0>.
\end{aligned}
$$

Averaging of spin variables implies the trace over spin indices, which is obviously equal to zero in our case. Therefore the spin orbital interaction does not contribute into the sum rule.

The sum rule for electric transitions coincides with that of the paper [16]:

$$
\begin{aligned}
\sum_{\nu}\left(E_{\nu}-E_{0}\right)\left(|<\nu| \hat{O}_{21}|0>|^{2}+|<\nu| \hat{O}_{2-1}|0>|^{2}\right) & =-<0\left|\left[\hat{O}_{21},\left[H, \hat{O}_{2-1}\right]\right]\right| 0> \\
& =e^{2} \frac{\hbar^{2}}{m} \frac{5}{4 \pi} Q_{00}(1+\delta / 3) .
\end{aligned}
$$

\section{References}

[1] R. R. Hilton "A possible vibrational mode in heavy nuclei", Int. Conf. on Nuclear Structure (Dubna, June 1976), unpublished.

[2] R. R. Hilton, Ann. Phys. (N.Y.) 214 (1992) 258

[3] T. Suzuki, D. J. Rowe, Nucl. Phys. A289 (1977) 461

[4] N. Lo Iudice, F. Palumbo, Phys. Rev. Lett. 41 (1978) 1532

[5] D. Zawischa, J. Phys. G: Nucl. Part. Phys. 24 (1998) 683

[6] N. Lo Iudice, La Rivista del Nuovo Cimento 23 (2000) N.9

[7] K. Heyde, P. von Neuman-Cosel and A. Richter, arXiv:1004.3429v1 [nucl-ex] (2010).

[8] U. Kneissl, H. H. Pitz, and A. Zilges, Prog. Part. Nucl. Phys. 37 (1996) 349.

[9] A. Richter, Prog. Part. Nucl. 34 (1995) 261.

[10] E. B. Balbutsev, L. A. Malov, P. Schuck, M. Urban, and X. Viñas, Phys. At. Nucl. 71 (2008) 1012. 
[11] E. B. Balbutsev, L. A. Malov, P. Schuck, and M. Urban, Phys. At. Nucl. 72 (2009) 1305.

[12] V. G. Soloviev, A. V. Sushkov, N. Yu. Shirikova and N. Lo Iudice, Nucl. Phys. A600 (1996) 155 ,

V. G. Soloviev, A. V. Sushkov, N. Yu. Shirikova, Phys. Rev. C53 (1996) 1022

[13] P. Ring and P. Schuck, The Nuclear Many-Body Problem (Springer, Berlin, 1980).

[14] D. A. Varshalovitch, A. N. Moskalev and V. K. Khersonski, Quantum Theory of Angular Momentum (Nauka, Leningrad, 1975).

[15] E. B. Balbutsev, Sov. J. Part. Nucl. 22 (1991) 159.

[16] E. B. Balbutsev, P. Schuck, Nucl. Phys. A. 720 (2003) 293;

E. B. Balbutsev, P. Schuck, Nucl. Phys. A. 728 (2003) 471.

[17] E. B. Balbutsev, P. Schuck, Ann. Phys. 322 (2007) 489. 